\title{
Overlapping influences shape motor activity during hasty sensorimotor decisions
}

\author{
Gerard Derosiere ${ }^{1,4,}{ }^{*}$, David Thura ${ }^{2}$, Paul Cisek $^{3}$, Julie Duque ${ }^{1}$
}

\begin{abstract}
Affiliations
1 Institute of Neuroscience, Laboratory of Neurophysiology, Université catholique de Louvain,Brussels, Belgium

${ }^{2}$ Lyon Neuroscience Research Center - Impact team - Inserm U1028 - CNRS UMR5292 - Lyon 1 University, Bron, France

${ }^{3}$ Department of Neuroscience, Université de Montréal, Montréal, QC H3T 1J4, Canada

${ }^{4}$ Lead Contact
\end{abstract}

\section{* Corresponding author contact details}

Email address: gerard.derosiere@uclouvain.be

Twitter: @GDerosiere 


\section{Summary}

Humans and other animals are able to adjust their speed-accuracy tradeoff (SAT) at will depending on the urge to act, favoring either cautious or hasty decision policies in different contexts. An emerging view is that SAT regulation relies on influences exerting broad changes on the motor system, tuning its activity up globally when hastiness is at premium. The present study aimed to test this hypothesis. Fifty subjects performed a task involving choices between left and right index fingers, in which incorrect choices led either to a high or to a low penalty in two contexts, inciting them to emphasize either cautious or hasty policies. We applied transcranial magnetic stimulation on multiple motor representations, eliciting motor evoked potentials (MEP) in nine finger and leg muscles. MEP amplitudes allowed us to probe activity changes in the corresponding finger and leg representations, while subjects were deliberating about which index to choose. Our data indicate that hastiness entails a broad amplification of motor activity, though this amplification was limited to the chosen side. On top of this effect, we identified a local suppression of motor activity, surrounding the chosen index representation. Hence, a decision policy favoring speed over accuracy appears to rely on overlapping processes producing a broad (but not global) amplification and a surround suppression of motor activity. The latter effect may help increasing the signal-to-noise ratio of the chosen representation, as supported by single-trial correlation analyses indicating a stronger differentiation of activity changes in finger representations in the hasty context.

\section{Keywords}

Motor cortex, action selection, speed-accuracy tradeoff, corticospinal excitability, urgency, motor-evoked potentials, decision-making. 


\section{INTRODUCTION}

From insects to rodents to primates, sensorimotor decisions are characterized by an inherent covariation between speed and accuracy, making the speed-accuracy tradeoff (SAT) a universal property of animal behavior ${ }^{1,2}$. Still, humans and other animals are able to adjust their SAT at will depending on the urge to act, favoring either hasty (i.e., high speed, low accuracy) or cautious (i.e., low speed, high accuracy) decision policies in different contexts. Given the importance of SAT regulation in the animal realm, and the deleterious impact of its disruption in major human diseases, such as in impulse-control disorders ${ }^{3-6}$, extensive research is being devoted to understanding its neural basis ${ }^{7-9}$.

Sensorimotor theories of decision-making postulate that decisions between actions arise, at least partly, from a competition between neural populations responsible for action execution in the motor system ${ }^{10-17}$. This theoretical view has prompted the field to investigate the motor system as a potential site for SAT regulation ${ }^{18-27}$. Consistently, motor activity appears to undergo influences pulling it upwards when the urge to act is high, in contexts calling for hasty decisions ${ }^{18,20,22,25-27}$. Furthermore, converging lines of evidence suggest that the source of this modulation may involve subcortical structures, especially the basal ganglia ${ }^{24,27-31}$ and the noradrenergic system ${ }^{20,22,32,33}$, which are known to exert broad influences on the motor cortex. Because of these two sets of findings, an emerging view in the field is that SAT regulation relies on influences exerting broad changes in the motor system ${ }^{20,22,25}$, tuning its activity up in a global manner when hastiness is at premium, irrespective of the neural population ultimately recruited for the action.

Global modulation represents a key candidate mechanism for how animals adjust their behavior in different SAT contexts, especially when considered in the light of computational models of decision-making ${ }^{7,34}$. In "drift-diffusion models", deliberation between actions involves an accumulation of evidence, which drives the build-up of neural signals coding for different actions towards a critical decision threshold in the motor system, and once one of them reaches this threshold, the related action is chosen and executed ${ }^{35-43}$. An alternative model suggests that sensory evidence is computed quickly, and the build-up of neural signals is primarily due to a growing "urgency signal" that pushes the system to reach the decision threshold even if evidence remains low $w^{45,47,52}$. While there is continued debate on whether neural activity build-up is primarily due to evidence accumulation versus urgency, all of these models suggest that control of SAT can be accomplished through a global motor amplification (i.e., in hasty contexts). This unique mechanism would explain how animals speed up their decisions and why they are more prone to make incorrect choices when they do so.

The explanatory power of the global modulation idea has contributed to its dissemination in the field of decision-making ${ }^{20}$. Yet, direct evidence for a contextdependent modulation of activity that is global across the motor system remains scarce in the SAT literature. In fact, if changes in motor activity have been interpreted through the lens of a global mechanism, the studies themselves were not designed to address directly the scope of modulatory changes per se, which would require considering different neural populations across the somatotopic map. Indeed, single-cell studies in monkeys only targeted one particular population (e.g., the arm area during reaching decisions $^{23,25}$ or eye areas during oculomotor decisions ${ }^{26,27}$ ), while 
electroencephalography studies in humans lacked the spatial resolution for tackling this critical issue ${ }^{20,22}$. A current challenge in the field is thus to characterize the scope of activity changes that may occur in different neural populations of the motor system during SAT regulation. Addressing this critical issue would provide insights into which structures may be at the origin of SAT-based motor changes and how neuromodulation of motor activity may be used to adjust SAT in impulse control disorders that are typically associated with hasty behaviors.

One fruitful approach to tackle this challenge is through the analysis of motorevoked potentials (MEPs), elicited by the application of single-pulse transcranial magnetic stimulation (TMS) over the primary motor cortex (M1) ${ }^{44,46}$. When applied over M1, TMS depolarizes populations of corticospinal neurons - often referred to as "motor representations" - that project down to specific body parts ${ }^{48,49}$, and generates MEPs in targeted muscles. Because corticospinal populations partly overlap in M1, TMS applied over one site can elicit MEPs in several muscles that are close together (e.g., several finger muscles). Importantly, neurons of the corticospinal pathway are under the influence of various subcortico-cortical circuits ${ }^{50}$. Hence, the amplitude of MEPs provides a population-specific readout of the net impact of these modulatory circuits on motor representations at the time of the stimulation ${ }^{51}$.

Here, we took advantage of these key TMS attributes to map the spatiotemporal features of modulations affecting the motor system during SAT regulation in humans. Fifty subjects performed a modified version of the "tokens task"3, involving choices between left and right index fingers. Incorrect choices led either to a high or to a low penalty in two different SAT contexts, inciting subjects to emphasize either cautious or hasty decision policies, respectively. We tested two groups of subjects in which TMS was applied at different stages of the decision-making task, either over the finger representations (TMS Finger subjects; bilateral M1 TMS with a double-coil procedure, eliciting simultaneous MEPs in three finger muscles on both sides) or over the leg representation (TMS Leg subjects; unilateral TMS with single-coil over left M1).

We focused on two main aspects of the MEP data. First, we considered the amplitude of MEPs from all finger and leg muscles over the course of the deliberation process in the two SAT contexts. This allowed us to assess the spatiotemporal features of motor excitability changes associated with SAT regulation in the task. Second, we considered the relationship between excitability changes shaping the chosen index finger and those occurring in the other finger muscles on the side of both the chosen and unchosen index fingers in the two contexts. To do so, we focused on data in the TMS Finger subjects, where we obtained simultaneous MEPs from six muscles (three on each side) in each trial. More precisely, we investigated the degree to which the trialby-trial MEP variation in the chosen index finger related to the trial-by-trial MEP variation in the other finger muscles. The rationale here was that a high positive correlation between the chosen index and the other finger muscles would indicate the operation of influences exerting a broad, common impact on their motor representations ${ }^{53-58}$, shaping MEPs in block. In contrast, a low or even a negative correlation would indicate the presence of influences affecting the chosen index representation in a more selective and differentiated way ${ }^{53,55,59}$. We compared the correlation values obtained in the hasty and the cautious contexts during deliberation.

Altogether, our data support the view that hastiness entails a broad amplification of motor excitability. As such, the hasty context was associated with particularly large 
MEPs, including in the leg muscles, though this effect was not entirely global as it was limited to the chosen side; it did not extend to muscles on the unchosen body side. Interestingly, on top of this effect, we also identified a local suppression of motor excitability, surrounding the index representation, also on the chosen side. Hence, a decision policy favoring speed over accuracy appears to rely on overlapping processes producing a broad (but not global) amplification and a surround suppression of motor excitability. The latter effect may help to increase the signal-to-noise ratio of the chosen representation, as also supported by the correlation analyses indicating a stronger differentiation of excitability changes between the chosen index and the other finger representations in the hasty relative to the cautious context. 


\section{RESULTS}

On each trial of the tokens task, 15 tokens jump one-by-one every $200 \mathrm{~ms}$ from a central circle to one of two lateral target circles. Here, subjects had to choose between left and right index finger key-presses depending on which lateral circle they thought would ultimately receive the majority of the tokens (Figure 1.A). They were free to respond at any time from Jump 1 to Jump 15 . Correct and incorrect choices led to rewards and penalties, respectively (Figure 1.B). The reward provided for correct choices decreased over the course of the trial, producing an increasing urge to decide. Most importantly, in one type of block, we sanctioned incorrect choices severely, with a penalty of -14 cents, emphasizing the need for cautiousness (cautious context). Conversely, the penalty provided for incorrect choices was only of -4 cents in the second block type, encouraging subjects to make hasty decisions (hasty context).

We exploited single-pulse TMS over M1 in two subgroups of subjects to quantify changes in motor excitability occurring in distinct representations, by probing MEPs in nine different muscles (Figure 1.C and Figure S1 in Supplemental Information). In TMS Finger subjects $(n=21)$, a double-coil approach was used to stimulate simultaneously the finger representations of both M1s. MEPs were recorded, concurrently in both hands, in an index, a thumb and a pinky muscle. The index muscle being the prime mover in the task, its MEPs allowed us to quantify motor excitability changes in a choice-relevant motor representation. The thumb and pinky muscles being not required in the task, their MEPs allowed us to assess excitability changes associated with choice-irrelevant representations that lie close by the prime mover representation in the motor system (i.e., in terms of somatotopy). In TMS Leg subjects $(n=22)$, we stimulated the left leg representation and recorded MEPs in three right leg muscles. These muscles being not required in the task, their MEPs allowed us to assess changes associated with choice-irrelevant representations that lie far from the prime mover in terms of somatotopy. Further, because the task required deciding between right and left index finger choices, MEPs could be classified according to whether they fell on the same side as the chosen index or on the side of the unchosen index, providing us with measures of excitability for each side in both the TMS Finger $_{\text {and }}$ the TMS Leg subjects. Finally, a subgroup of No-TMS subjects $(n=7)$ performed the task without stimulation, allowing us to control for any effect of TMS on decision behavior. 
A.

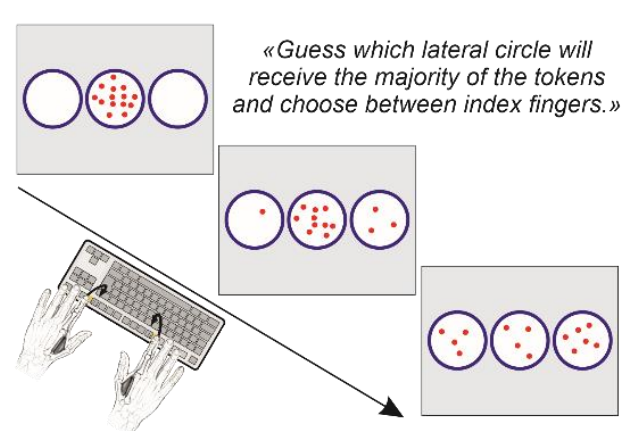

B. SAT contexts

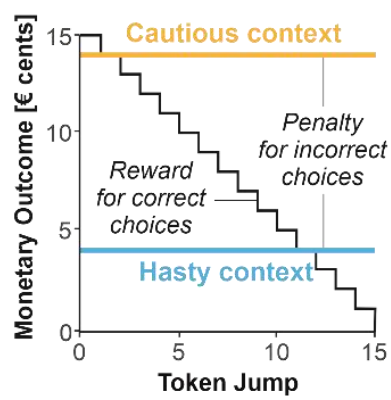

C.

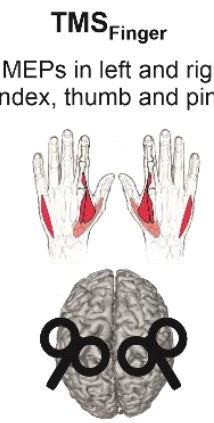

Motor Excitability Mapping

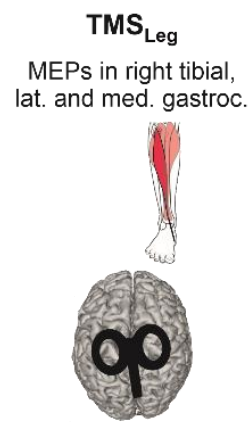

Figure 1: Tokens task, SAT contexts and motor excitability mapping. A. Tokens task. Subjects had to choose between left or right index finger key-presses depending on which lateral circle they thought would ultimately receive the majority of the tokens. B. SAT contexts. The reward provided for correct choices decreased over the course of the trial, producing an increasing urge to decide. Most importantly, the use of a low penalty ( -4 cents; blue) promoted hasty choices (hasty context), while a high penalty (-14 cents; yellow) fostered cautious choices (cautious context). In both contexts, a low penalty ( -4 cents) was provided when subjects did not respond before Jump ${ }_{15}$ (not represented here). C. Motor excitability

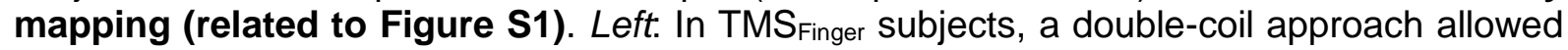
us to elicit MEPs in index, thumb and pinky muscles of both hands. In TMS Leg subjects, MEPs were recorded in three right leg muscles. MEPs recorded these nine muscles were of reliable amplitude and were reproducible across sessions (see Figure S1). Right: MEPs obtained in these different muscles allowed us to quantify excitability changes associated with a choicerelevant motor representation (i.e., the index finger representation), choice-irrelevant representations that lie close by the choice-relevant one (i.e., the thumb and pinky representations), and choice-irrelevant representations that lie far from the choice-relevant one (i.e., the leg representations). Further, classifying MEPs according to whether they fell on the same side as the chosen index or on the side of the unchosen index allowed us to measure excitability of the motor system on both the chosen and the unchosen side.

\section{Subjects regulated their decision behavior depending on context}

To highlight the presence of a SAT in our task, we regressed individuals' percentages of correct choices (i.e., accuracy) against their decision times (DTs) using a permutation-based correlation (Nsubjects $=50$, Nermutations $=1000)$. As expected, this analysis showed a significant positive correlation between DTs and accuracy, with subjects presenting the fastest DTs being also the least accurate, both in the hasty and 
in the cautious contexts $(R=.72$ and $R=.68$, respectively, both $p$-values $<.0001$; Figure 2.A). Most importantly, the subjects' distribution appeared shifted in the hasty relative to the cautious context, supporting a change in SAT (e.g., see distributions in the margins of Figure 2.A). Indeed, a between-context comparison revealed that both DTs and accuracy were significantly lower in the hasty context $(\mathrm{t} 49=-8.42, \mathrm{p}<.0001$, Cohen's $d=1.191$ and $t 49=-11.26, p<.0001$, Cohen's $d=1.593$, respectively; Figure 2.B and $C$ ). Altogether, these findings show that subjects regulated their SAT in accordance with our expectations, favoring a hasty policy when the context involved a low penalty and emphasizing cautiousness when a high penalty was at stake.

Next, we tested whether subjects exhibited changes in the level of urgency from one context to another. To do so, we extracted urgency functions using a previously described computational analysis of decision behavior ${ }^{60,61}$. As predicted by timevarying models of decision-making $20,52,62,63$, we found that urgency increased significantly as time elapsed during deliberation, both in the hasty and in the cautious contexts (i.e., t-tests against 0 on slope values: $t 49=14.58$, Cohen's $d=2.069$ and $t 49$ $=19.0$, Cohen's $d=2.667$, respectively, Bonferroni-corrected $p$-values < .0001; Figure 2.D). Most importantly, while the slope of the functions did not differ significantly between contexts ( $\mathrm{t} 49=-0.82, \mathrm{p}=.419$, Cohen's $\mathrm{d}=0.124$ ), the intercept was significantly higher in the hasty than in the cautious context $(\mathrm{t} 49=7.42, \mathrm{p}<.0001$, Cohen's $d=1.050)$. Together, these findings indicate that the level of urgency was higher in the hasty than in the cautious context at the start of the deliberation period and that this difference persisted throughout that period.

Finally, we analyzed the DTs, accuracy, as well as the slope and intercept of the urgency functions while considering the TMS subgroup (i.e., TMS Finger, TMS Leg, NoTMS subjects) as a categorical predictor in our analyses (i.e., using ANOVAs). We did not find any significant effect of the TMS subgroup on the behavioral data, nor of its interaction with the factor CONTEXT (i.e., hasty vs cautious context; Figure S2). Further, a Bayes Factor analysis provided evidence for a lack of effect of the subgroup on all of these behavioral data. In fact, the three subgroups presented very similar effects of context on decision behavior. This indicates that the application of TMS over the finger or leg representations did not perturb SAT regulation in this task. 

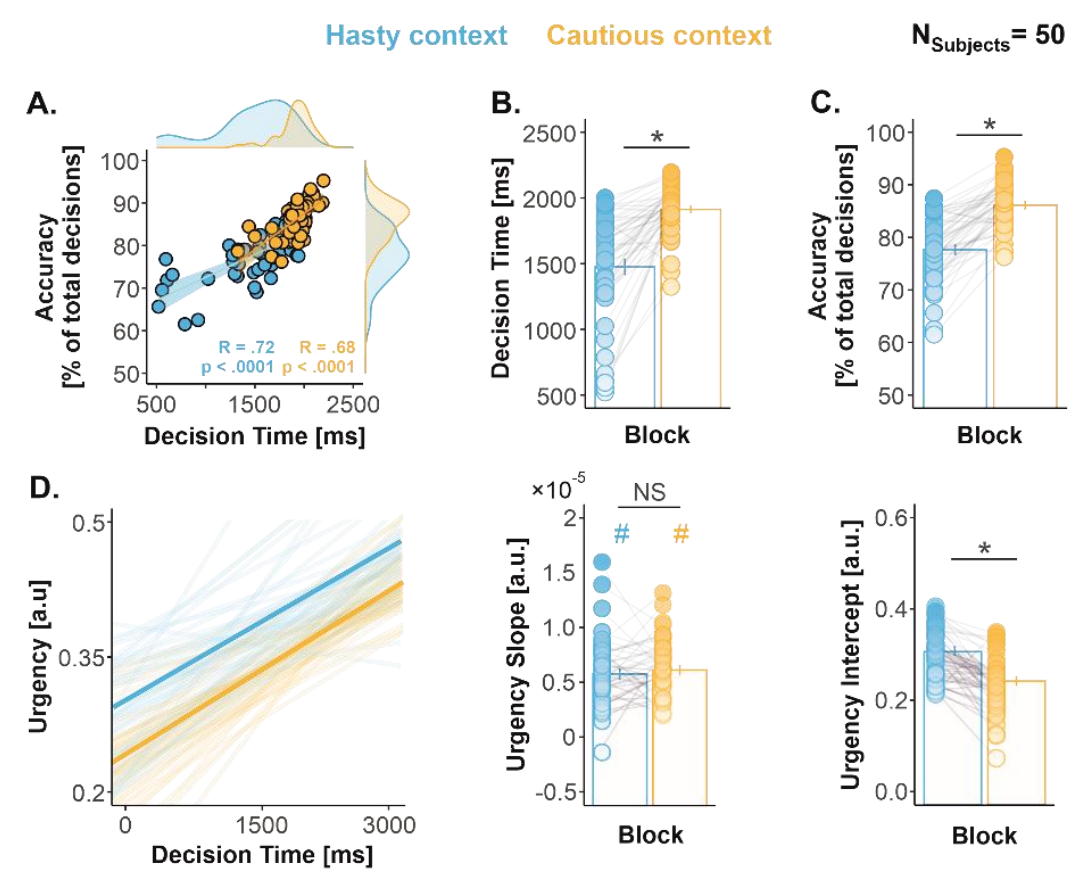

Figure 2 (related to Figure S2): Subjects shifted their speed-accuracy tradeoff in the hasty relative to the cautious context. A. Speed-Accuracy relationship. A permutationbased correlation $\left(N_{\text {Permutations }}=1000\right.$ ) revealed a significant positive correlation between individuals' DTs and accuracy, present in both contexts. As expected, the fastest subjects were also the least accurate, highlighting the presence of a speed-accuracy tradeoff in the task. B. Decision times. Subjects presented significantly faster DTs in the hasty compared to the cautious context. C. Decision accuracy. Accuracy was significantly lower in the hasty context. D. Urgency functions. While the slope of the functions was comparable in the hasty and the cautious contexts (middle panel), the intercept was significantly higher in the former context. As a result, the level of urgency was higher in the hasty context throughout the deliberation period. ${ }^{*}$ : significant effect of context at $p<.05$. Error bars represent 1 SEM.

\section{Motor excitability globally increased as time elapsed during deliberation}

To assess the dynamics of motor excitability changes over the decision period, we applied TMS in $90 \%$ of trials, at one of four possible timings during the task: at Jump 1 , Jump 4 and Jump7, as well as at baseline (i.e., between the trials; Figure 3.A). In order to capture excitability changes related to deliberation, we selected trials in which responses occurred at least $150 \mathrm{~ms}$ after Jump 7 and up to Jump 15 . Further, to prevent MEP amplitudes from being affected by the difference in decision speed between each context, we homogenized the reaction time (RT) distributions across contexts by selecting trials through a RT-matching procedure ${ }^{20}$ (Figure S3). Following this procedure, we had to exclude 2 out of the 21 TMS Finger subjects and 6 out of the 22 TMS Leg participants, as they ended up with less than 8 trials on average per timing and

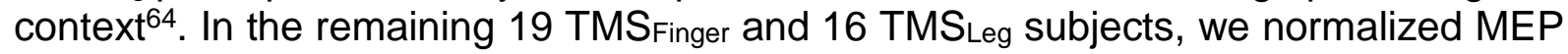
amplitudes obtained at Jump 1 , Jump 4 and Jump 7 as a percentage of baseline ${ }^{65}$ for each motor representation and for both the chosen and the unchosen sides.

Normalized MEP amplitudes displayed a main effect of TIMING in TMS Finger subjects (i.e., Jump 1 vs Jump 4 vs Jump 7 in a repeated-measures [rm]ANOVA). This effect was significant for both the chosen (Greenhouse-Geiser [GG]-corrected: $F_{1.4,25.4}=10.610$, 
$\mathrm{p}=.001$, partial $\eta^{2}=.371$; Figure 3.A) and the unchosen side MEPs (GG-corrected: $F_{1.5,26.3}=8.3024, p=.003$, partial $\eta^{2}=.283$; Figure 3.B). Tukey's HSD post-hoc tests revealed that amplitudes were significantly larger at Jump compared to Jump $_{1}$ and

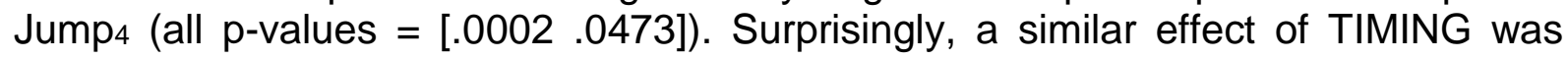
observed in TMS Leg subjects, with MEPs growing over time on both the chosen $\left(F_{2,30}\right.$ $=10.206, p=.0007$, partial $\eta^{2}=.405$; Figure 3.A) and the unchosen side $\left(F_{2,30}=\right.$ 21.716, $\mathrm{p}<.0001$, partial $\eta^{2}=.591$; Figure 3.B). Here, post-hoc tests revealed that amplitudes were larger at Jump 4 and Jump than at Jump 1 (all p-values $=[.0012$ $.0001])$. Overall, these findings indicate that motor excitability exhibited a global increase as time elapsed during the decision process. This time-dependent effect not only concerned choice-relevant representations, but also choice-irrelevant ones, and even those lying far away within the motor system (leg), on both the chosen and the unchosen sides. 


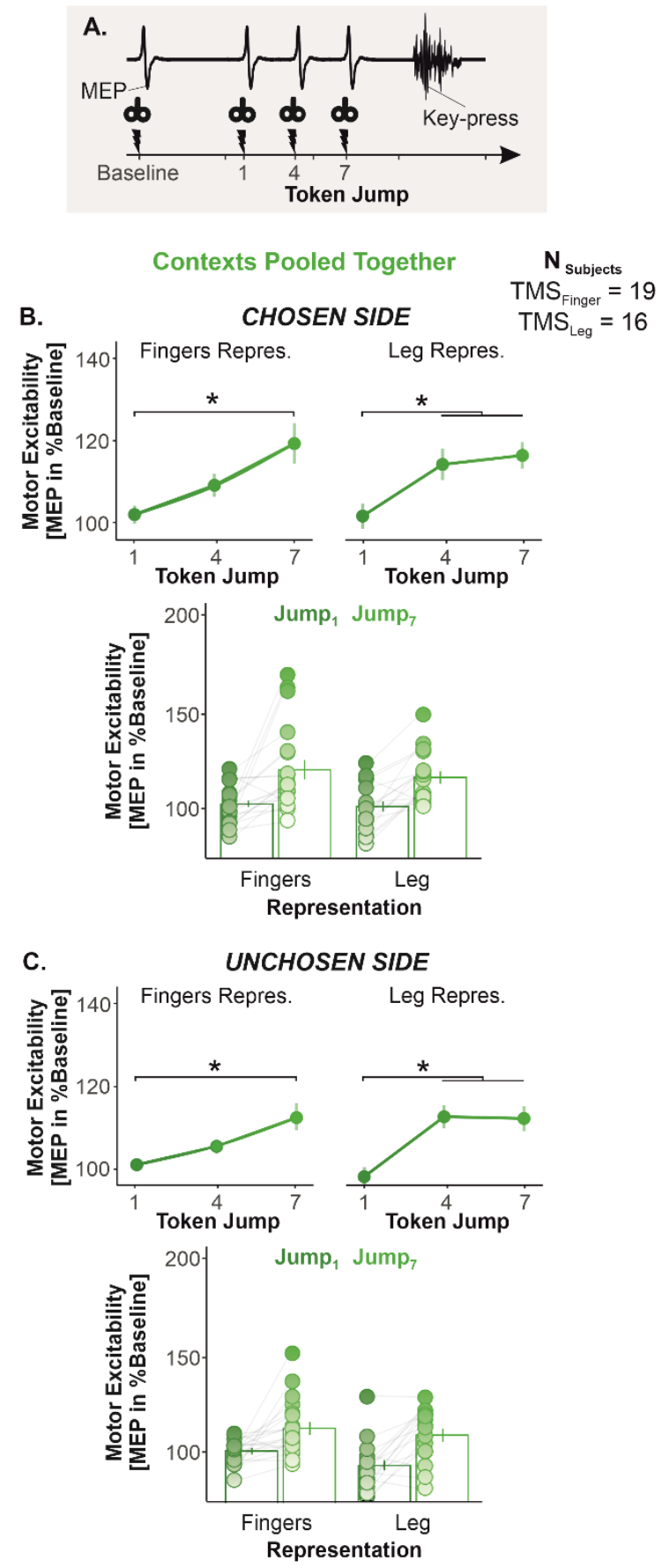

Figure 3 (related to Figure S3): Motor excitability globally increased as time elapsed during deliberation. A. TMS timings. We applied TMS at four timings during the task: at baseline (i.e., between the trials) and at Jump 1 , Jump 4 and Jump 7 during the decision period. B. Main effect of TIMING on motor excitability on the chosen side. To highlight the main effect of TIMING, both SAT contexts were pooled together. Further, the data obtained for the index, the thumb and the pinky representations (top left) were averaged for the figure as well as the data obtained for the three leg representations (top right). The bar graph at the bottom displays the individual data points, as obtained at Jump ${ }_{1}$ (dark green) versus Jump (light $^{2}$ green). Overall, this representation shows that a large proportion of subjects exhibited an increase in motor excitability from Jump ${ }_{1}$ to Jump ${ }_{7}$, both in the finger and in the leg representations. C. Same as B. for the unchosen side. Error bars represent 1 SEM. * significant effect of timing at $p<.05$. 


\section{Hastiness relied on a broad amplification and a surround suppression of motor excitability on the chosen side during deliberation}

Importantly, MEP amplitudes also showed a significant effect of CONTEXT on the chosen side, which varied as a function of the TIMING and of the finger REPRESENTATION in TMS Finger subjects (CONTEXT*'TIMING*REPRESENTATION interaction: $F_{4,72}=3.63, p=.009$, partial $\eta^{2}=.168$; see Figure 4.A). Tukey's HSD posthoc tests revealed that the MEPs obtained in the index muscle were significantly larger in the hasty than in the cautious context at Jump $7(p=.021)$, indicating an amplification of motor excitability in the choice-relevant representation. Notably, TMS Leg subjects also displayed a significant main effect of CONTEXT on the chosen side $\left(F_{1,15}=4.65\right.$, $p=.047$, partial $\eta^{2}=.237$; see Figure 4.A, right panel), with leg MEPs being larger in the hasty than in the cautious context, indicating that the amplification of motor excitability also affected the leg representations. This effect was reproducible across the three investigated leg muscles (non-significant GG-corrected CONTEXT*TIMING*REPRESENTATION interaction $F_{2.4,36.6}=1.54, p=.226$, partial $\eta^{2}$ $=.093$, see Figure S4). Hence, on the chosen side, MEPs were significantly larger in the hasty than in the cautious context and this effect not only concerned the choicerelevant (chosen) muscle, but also choice irrelevant leg muscles that lie far from the prime-mover. Besides that, post-hoc tests performed on the TMS Finger subjects' data (i.e., following the CONTEXT*TIMING*REPRESENTATION interaction mentioned above) also revealed an additional effect that concerned specifically the thumb and pinky muscles on the chosen side. Here, MEPs were significantly smaller in the hasty than in the cautious context at Jump $7 \mathrm{p}=.013$ and .0247 for the thumb and pinky fingers, respectively), suggesting thus a suppression of excitability in the surrounding choice-irrelevant representations. Altogether, these data indicate that the SAT shift observed in the hasty context was associated with the occurrence of two overlapping modulatory changes on the chosen side: a broad amplification expanding to remote choice-irrelevant representations, and a local suppression of choice-irrelevant representations surrounding the choice-relevant one.

Interestingly, these modulatory changes did not involve the unchosen side. As evident in Figure 4.B, MEP amplitudes obtained there did not show any significant effect of CONTEXT $\left(F_{1,18}=0.57, p=.457\right.$, partial $\eta^{2}=0.031$ and $F_{1,15}=1.06, p=.318$,

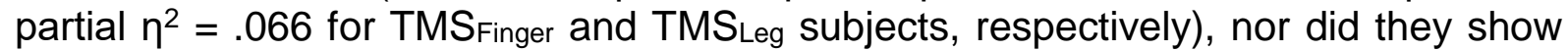
any CONTEXT ${ }^{\star}$ TIMING $\left(F_{2,36}=0.01, p=.987\right.$, partial $\eta^{2}=6.88 \times 10^{-5}$ and $F_{2,30}=0.65$, $\mathrm{p}=.529$, partial $\left.\eta^{2}=.041\right)$, CONTEXT*REPRESENTATION $\left(\mathrm{F}_{2,36}=.06, \mathrm{p}=.935\right.$, partial $\eta^{2}=.004$ and $F_{2,30}=0.589, p=.561$, partial $\eta^{2}=.037$ ) or CONTEXT*TIMING*REPRESENTATION interaction $\left(F_{4,72}=1.22, p=.310\right.$, partial $\eta^{2}$ $=.063$ and $F_{4,60}=0.47, p=.756$, partial $\left.\eta^{2}=.030\right)$. BFs for all of these effects ranged between 7.82 and 492.78, providing further evidence for a lack of effect of context on the unchosen side ${ }^{66}$. Hence, while the chosen side undergoes a broad amplification adding up to a local suppression when decisions have to be fast, representations on the unchosen side remain largely unaffected by the context.

Based on these data, we computed spatiotemporal maps to provide an integrative view of motor excitability changes occurring during the course of deliberation (i.e., for each stimulation time) in each context (Figure 4.C). To this aim, we considered altogether the MEPs obtained for the index, thumb, pinky and leg representations on 
the side of both the chosen and unchosen index fingers (i.e., 8 representations), in each context. The 8 traces were spatially arranged according to M1 somatotopy (i.e., from lateral to medial: thumb, index, pinky, leg) and a linear interpolation was performed to estimate excitability changes between each representation. Two spatiotemporal maps were obtained (one for each context) and a between-context contrast map was finally computed (i.e., hasty minus cautious context). The difference map highlights the increase in excitability (in green) for the index of the chosen side, expanding to leg representations, as well as the suppression (in red) occurring in the surrounding thumb and pinky representations (Figure 4.C, right panel). 


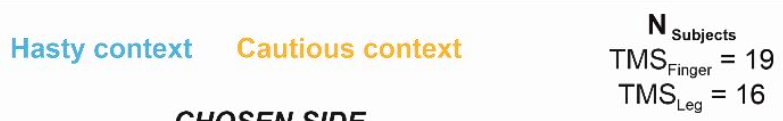

A.
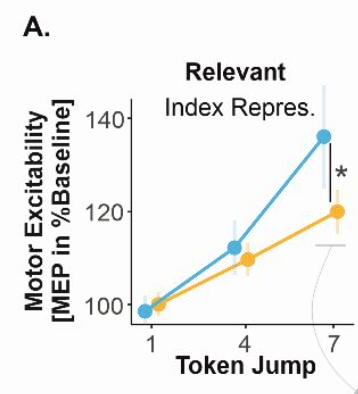

Thumb Repres. $\quad$ Pinky Repres.

Irrelevant Far

Leg Repres.
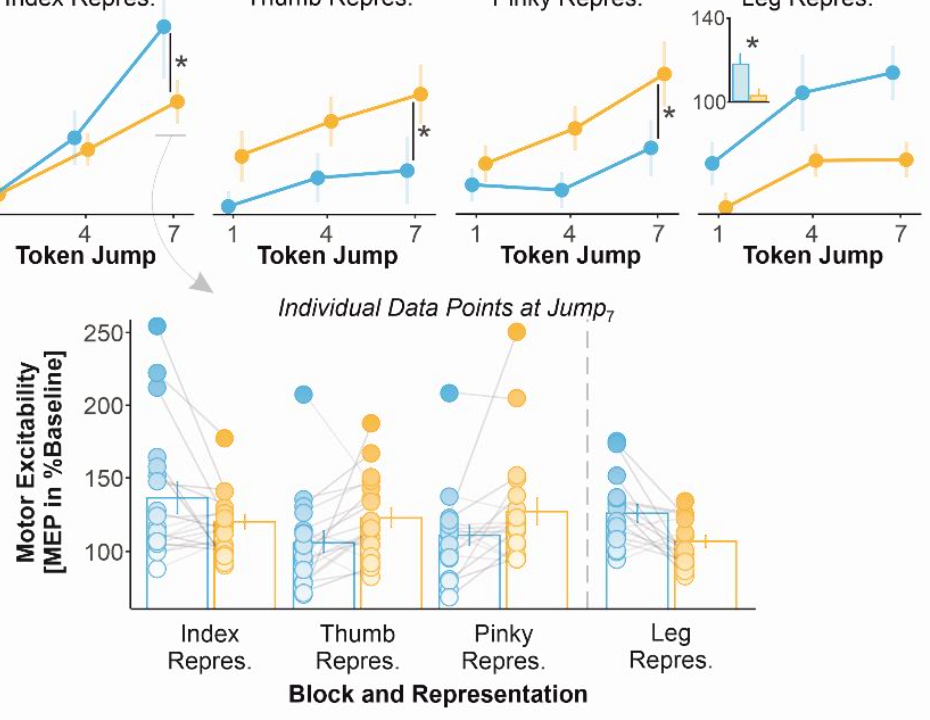

B.
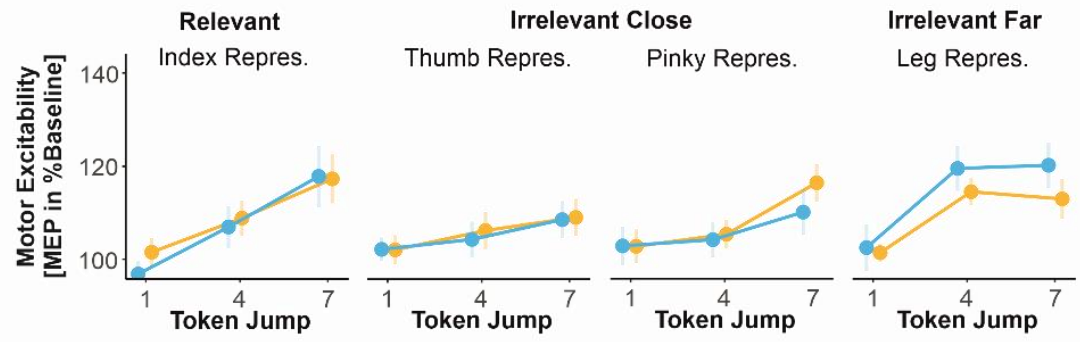

c.

SPATIOTEMPORAL MOTOR MAPS

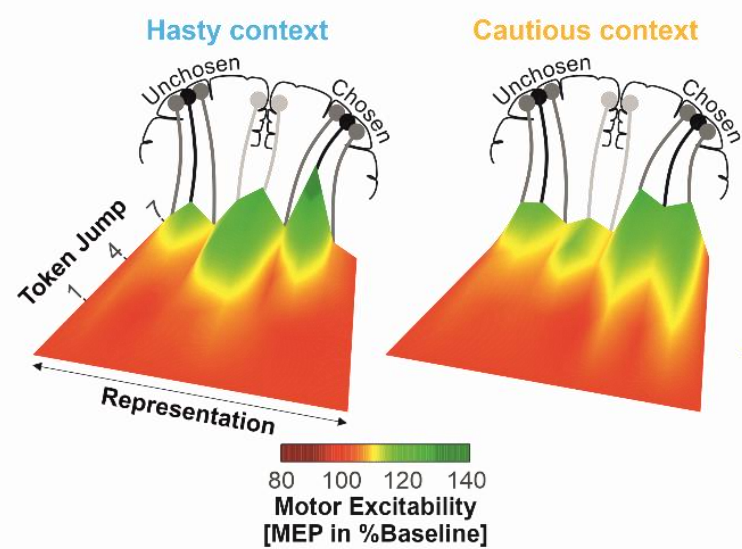

Between-Context Contrast

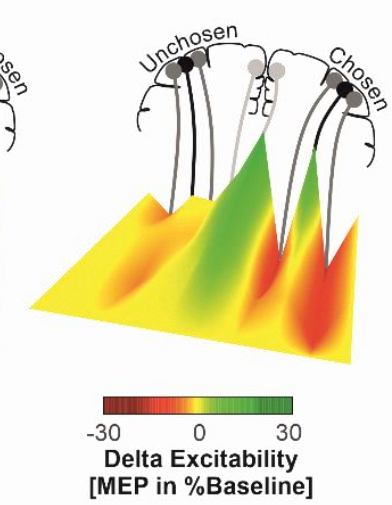

- Index Repres.: Relevant -Thumb \& Pinky Repres.: Irrelevant Close

- Leg Repres.: Irrelevant Far

Figure 4 (related to Figure S4): Hastiness relied on a broad amplification and a surround suppression of motor excitability on the chosen side. A. Effect of CONTEXT on motor excitability on the chosen side. The top graphs show excitability changes occurring over the decision period in the hasty and cautious contexts (blue and yellow traces, respectively). Given that the effect of context was reproducible across the three leg representations (see Figure S4), the MEP data have been pooled together (right panel). The inset in the right panel denotes 
the main effect of context on the leg region. The bar graph at the bottom displays individual data points as obtained at Jump ${ }_{7}$, for each context and each representation. Error bars represent 1 SEM.*: significant effect of context at $p<.05$. B. Same as A. for the unchosen side. C. Spatiotemporal motor maps. We computed spatiotemporal maps to provide an integrative view of motor excitability changes occurring during the course of deliberation in each context (see main text). To this aim, we considered altogether the MEPs obtained for the index, thumb, pinky and leg representations of the chosen and unchosen sides, and we arranged them spatially according to M1 somatotopy. One spatiotemporal map was obtained for each context and a between-context difference map was finally computed (i.e., hasty minus cautious context). The difference map (right panel) highlights the increase in excitability in the index and leg representations of the chosen side (right side of the map, green, positive values) as well as the surround suppression occurring in the thumb (more lateral) and pinky (more medial) representations (red, negative values). Besides, no noticeable between-context difference emerged on the unchosen side (left side of the map, yellow values).

\section{Hastiness did not affect baseline activity}

The data presented in Figure 4 highlight the effects of context on motor excitability during deliberation. Next, we wanted to assess whether context also altered excitability outside the deliberation period, when subjects were resting between trials. To do so, MEP amplitudes obtained at the baseline timing (see Figure 3.A) were normalized with respect to MEPs recorded at rest, outside of the task ${ }^{65}$. We did not apply any RTmatching procedure on these data, as the baseline timing was deemed too far from the deliberation period to be affected by any between-context difference in decision speed. This allowed us to include every subject in the analysis (i.e., 21 TMS Finger subjects and 22 TMS Leg subjects).

Interestingly, we did not find any significant effect of the factor CONTEXT (TMS Finger subjects: $F_{1,20}=1.14, p=.297$, partial $\eta^{2}=.054$; TMS Leg subjects: $F_{1,21}=0.59, p=$ .451 , partial $\eta^{2}=.027$ ) nor was there any interaction with the factor REPRESENTATION (TMSFinger subjects: $F_{2,40}=1.49, p=.236$, partial $\eta^{2}=.069$; TMSLeg subjects: and $F_{2,42}=2.17, p=.127$, partial $\eta^{2}=.093$ ) on baseline MEPs (Figure 5). Further, a Bayes factor analysis provided substantial evidence for a lack of effect of CONTEXT on these data (Bayes factors $=4.41$ and 3.55, for TMS Finger and TMS Leg subjects, respectively) and of the CONTEXT*REPRESENTATION interaction (Bayes factors $=9.53$ and 4.93 ). Altogether, these results indicate that the effect of context on motor excitability was restricted to the deliberation period, leaving baseline activity unaffected.

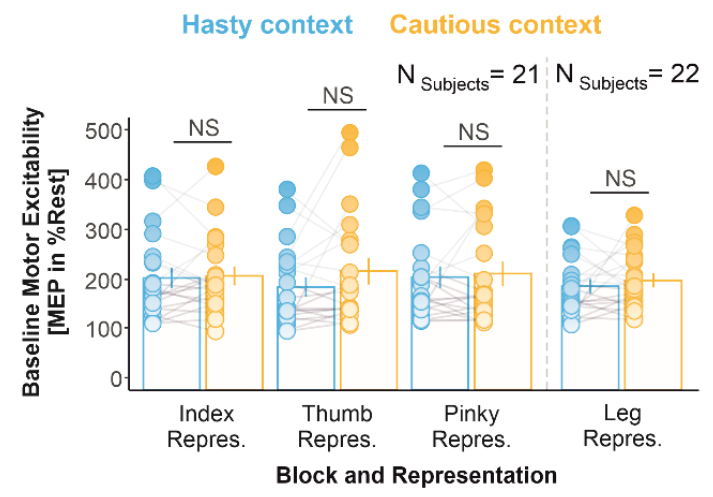

Figure 5: Hastiness did not affect baseline activity. NS denotes the lack of significant difference between both contexts. Error bars represent 1 SEM. 


\section{Hastiness was associated with a decorrelation of the chosen index and the other finger representations during deliberation}

To further characterize the impact of context at Jump7 (Figure 4.A), we quantified, in each context, the relationship between trial-by-trial MEP variations in the chosen index and in the 5 other fingers using Pearson's correlations (Figure 6.A). We adopted a permutation-based analysis ${ }^{67}$ to estimate statistical significance of each of the 10 correlations (Nermutations $=1000$; Bonferroni-corrected significance threshold at $p=$ .005). Then, for each of the 5 pairs of muscles, we compared the strength of the correlation in the hasty relative to the cautious context using the Fisher's $Z$ test ${ }^{68}$ (Bonferroni-corrected significance threshold at $p=.01$ ).

Overall, MEPs of the chosen index correlated positively with MEPs of the other fingers, whether on the chosen or unchosen side (Figure 6.A and Figure S5). However, while all of the 5 correlations were significant in the cautious context (permutationbased $p$-values $=[00001.005])$, only 1 was significant in the hasty context ( $p$-values = $.00001, .007, .035, .139$ and .311; Figure 6.B), suggesting a weaker trial-by-trial relationship in excitability changes between the chosen index and the other fingers in the latter context. Consistently, the Fisher's $Z$ test revealed that the strength of the correlation was often significantly weaker in the hasty than in the cautious context (see Figure 6.A). This was true when considering the link on the chosen side with the pinky $(R=.10 \pm .09$ vs. $R=.37 \pm .08$, respectively: Fisher's $Z=4.34, p=.0001)$, and on the unchosen side with the index $(R=.07 \pm .09$ vs. $R=.25 \pm .13$, respectively: Fisher's $Z$ $=2.87, p=.002$ ) and the thumb (though the effect of context was marginal here: $R=$ $.04 \pm .09$ vs. $R=.17 \pm .09$ : Fisher's $Z=2.01, p=.044)$. Altogether, these data indicate that hastiness involves a decoupling of excitability in the chosen finger representation with respect to the other finger representations, which may help enhancing the signalto-noise ratio of the chosen representation ${ }^{54}$. 


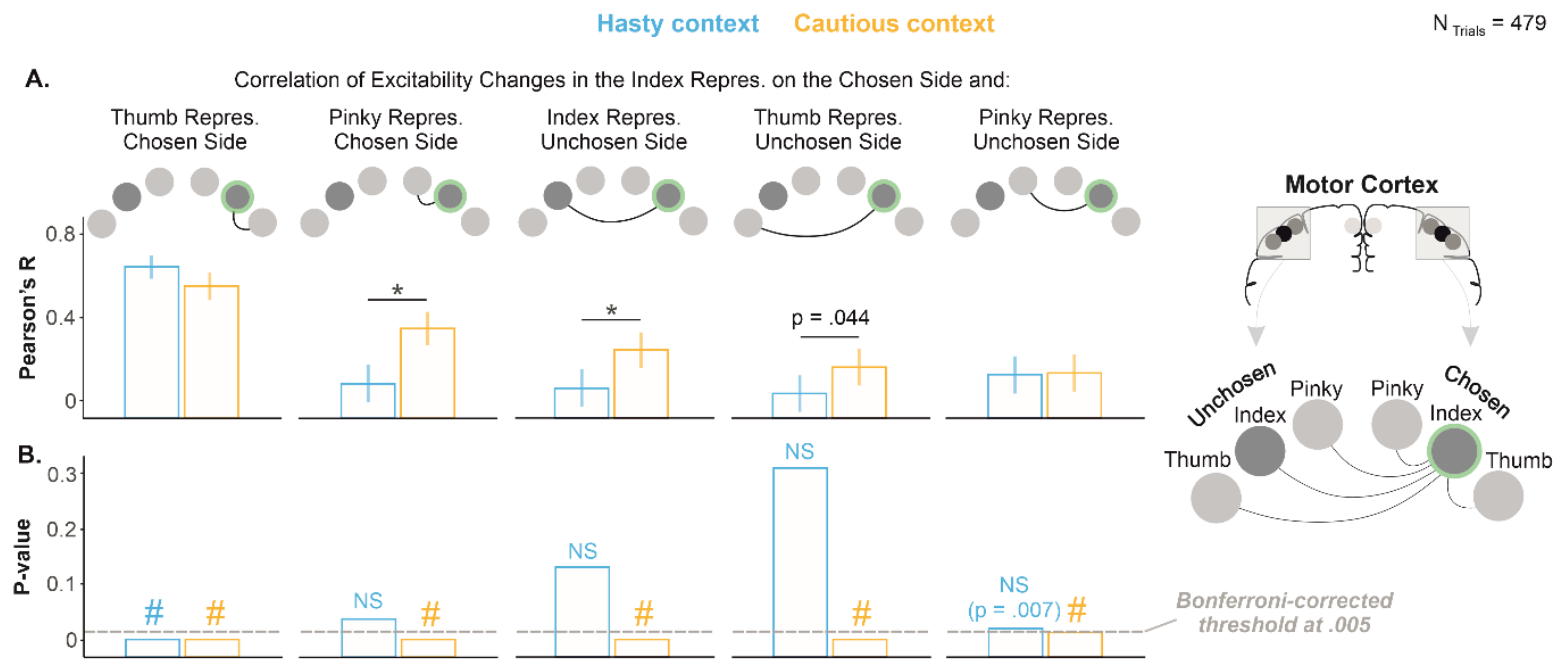

c.
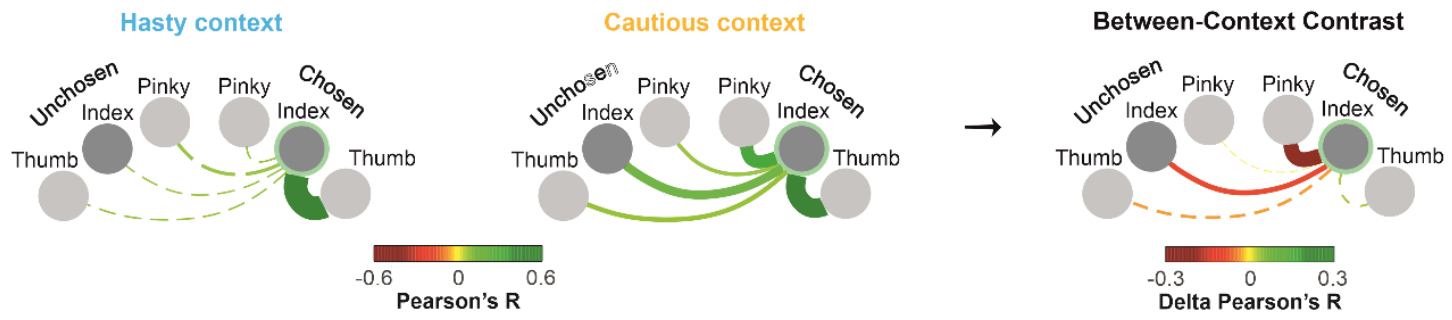

Figure 6 (related to Figure S5): Hastiness was associated with a decorrelation of excitability changes between the chosen index and the other finger representations during deliberation. A. Pearson's R-values. Error bars represent $95 \% \mathrm{Cl}$. * indicates a significant difference between R-values at $p<.01$ (Bonferroni-corrected threshold), detected using Fisher's Z test. B. Permutation-based p-values. \# indicates a significant correlation at $p<.005$ (Bonferroni-corrected threshold). C. Network plots. The color and thickness of the lines in the left and middle panels represent the strength of the correlations, as indexed by Pearson's R-values. Solid and dashed lines represent significant and non-significant correlations, respectively, as determined using the permutation-based procedure. In the right panel, the color and thickness of the lines represent the difference in R-values between the cautious and the hasty context. . Solid and dashed lines represent significant and nonsignificant correlations, respectively, as determined using Fisher's Z test.

\section{Movement vigor was unaffected by elapsed time and hastiness}

Given the known impact of movement vigor on motor activity $25,69,71$, we investigated EMG activity to test whether any change in vigor could have contributed to the changes in MEP amplitude observed in our task. To this aim, we exploited the EMG signals recorded in the moving hand of TMS Finger subjects (i.e., in the index, thumb and pinky muscles) and considered the voluntary contraction preceding the key-press in the two contexts. For each response provided, we rectified the signal and extracted the peak amplitude as a proxy of movement vigor ${ }^{22,70}$. To investigate the effect of elapsed time on this variable in each context, we split the trials into two subsets according to whether they were associated with short or long RTs, using a median-split procedure (RTshort and RTLong trials, respectively; Figure S6). Further, to prevent EMG peak amplitude from being affected by the difference in decision speed between each context, we homogenized the RT distributions across contexts through a RT-matching 
procedure ${ }^{20}$, both for RT short and RT Long trials. Following this procedure, we had to exclude 1 out of the 21 TMS Finger subjects, as she/he ended up with no trial in a specific condition (N $\mathrm{N}_{\text {subjects }}=20$ ).

As evident in Figure 7 (see also Figure S6), the analysis of EMG peak amplitude did not show any significant effect of CONTEXT $\left(F_{1,19}=.007, p=.934\right.$, partial $\eta^{2}=$ .003 ) or RTLength $\left(F_{1,19}=0.09, p=.763\right.$, partial $\left.\eta^{2}=.004\right)$. There was also no CONTEXT*RTLENGTH $\left(\mathrm{F}_{2,36}=.86, \mathrm{p}=.365\right.$, partial $\left.\eta^{2}=.043\right)$ or CONTEXT*RTLENGTH*MUSCLE interaction (GG-corrected: $F_{1.1,20.1}=1.81, p=.193$, partial $\eta^{2}=.087$ ). Bayes factors for all of these effects ranged between 7.07 and 7.32 , providing evidence for a lack of effect of elapsed time and context on EMG peak amplitude. Hence, the time- and context-dependent changes in motor excitability observed in our task cannot be accounted for by variations in movement vigor.

Hasty context Cautious context

A.
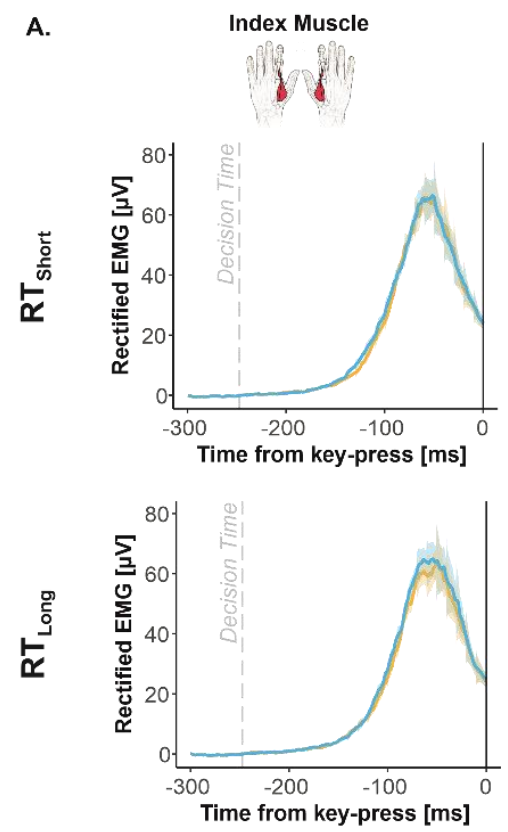

B.

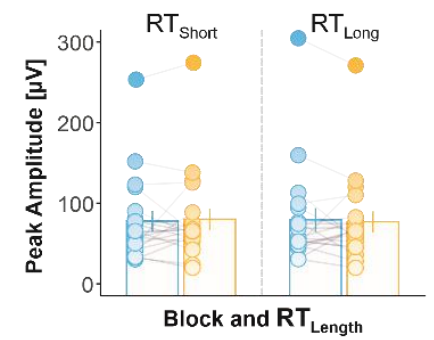

Thumb Muscle
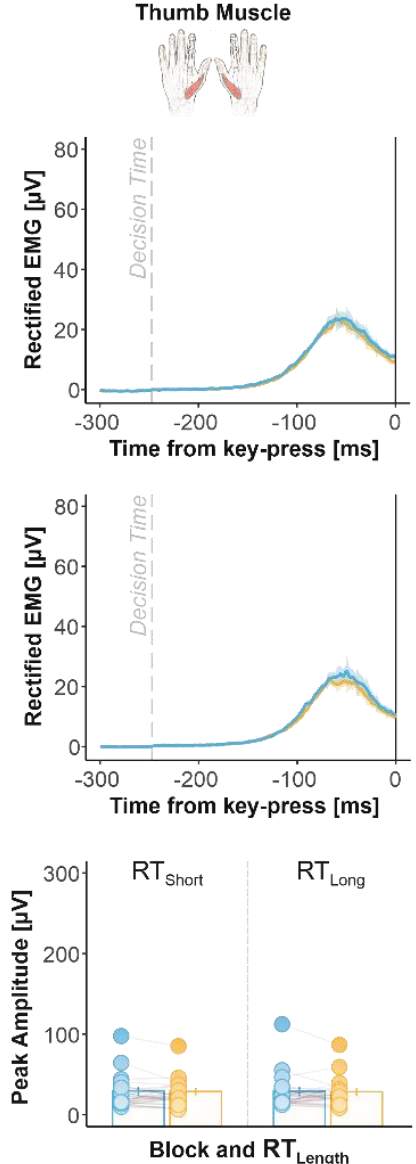

$\mathrm{N}_{\text {Subjects }}=\mathbf{2 0}$
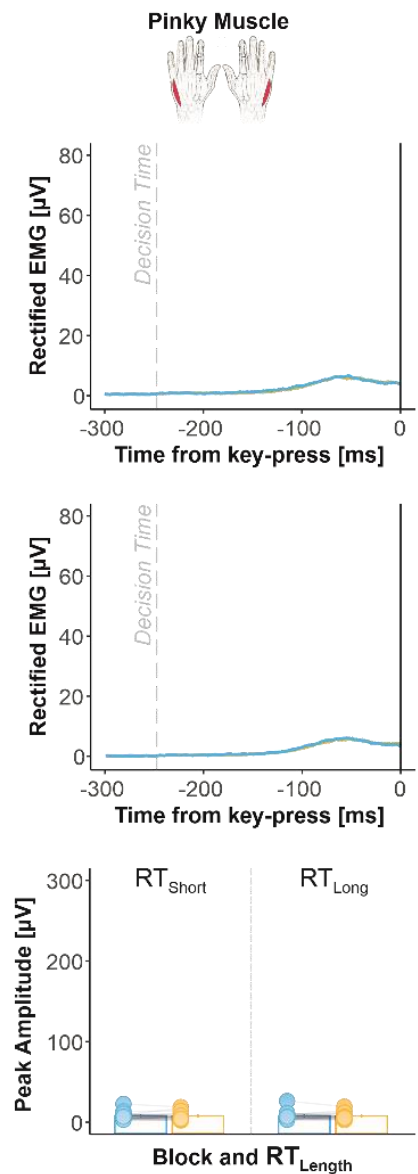

Figure 7 (related to Figure S6): Movement vigor was unaffected by elapsed time and hastiness. A. Group-averaged rectified EMG activity. Shaded error bars represent 1 SEM. The vertical dotted line indicates the estimated DT (see Methods). B. Group-averaged peak amplitude. Error bars represent 1 SEM. Overall, EMG activity was comparable for RT Short and $\mathrm{RT}_{\text {Long }}$ as well as across contexts in each of the three muscles. 


\section{DISCUSSION}

The goal of the present study was to test the hypothesis that SAT regulation relies on a global modulation of motor activity during sensorimotor decisions ${ }^{20,22,25}$. Subjects performed a task involving choices between left and right index fingers, in which incorrect choices led either to a high or to a low penalty in two contexts, inciting them to emphasize either cautious or hasty decision policies, respectively. We applied TMS on different motor representations in M1, eliciting MEPs in multiple finger and leg muscles at different stages of the decision-making task. MEP amplitudes allowed us to probe activity changes in the corresponding finger and leg representations, while subjects were deliberating about which index finger to choose.

Overall, participants made faster but less accurate choices when the context involved a low penalty, relative to when the penalty was high. Furthermore, a computational analysis of the behavioral data revealed that subjects exhibited a higher level of urgency in the low penalty context. The latter analysis also showed that urgency grew as time elapsed during the deliberation period, replicating previous findings in the literature ${ }^{20,52,62,63}$. Altogether, our decision data indicate that reducing the cost of incorrect choices increased participants' urge to act, leading them to shift their SAT from a cautious to a hasty decision policy.

The SAT shift observed in the hasty context was associated with a broad amplification of excitability on the chosen side during deliberation, altering both the choice-relevant (index) representation and remote choice-irrelevant (leg) representations. Importantly though, this modulation did not globally impact the motor system, leaving the unchosen side unaffected. Hence, these results do not support the idea of a global modulation of motor activity across contexts. Rather, they suggest the existence of broad neural sources pushing up motor activity unilaterally when context calls for hasty decisions, ensuring a faster rise-to-threshold of neural activity in the chosen hemisphere. Given their strong ipsilateral projections to the motor cortex ${ }^{72}$ and their known involvement in SAT regulation $24,28-30$, the basal ganglia represent a potential candidate for this unilateral, broad amplification, a hypothesis worthy of further investigation.

Interestingly, the broad amplification was restricted to the deliberation period and did not affect baseline activity. This result may appear to be at odds with previous findings of the literature, showing upward shifts in baseline activity in hasty contexts ${ }^{1,25,31}$. However, these so-called baseline shifts are most often observed right before the decision period ${ }^{25,31}$, while baseline measures were probed long before the start of the decision period in the present study (i.e., $1300 \mathrm{~ms}$ before the first token jump). As such, the impact of context on baseline activity may depend on the state of motor preparation, becoming stronger as the decision period - and therefore the need to act - draws nearer. In line with this interpretation, motor excitability was already amplified at the beginning of the decision period (i.e., at Jump 1 ) in the leg representations. Alternatively, it is possible that a fraction of corticospinal cells showed an amplification of activity at our baseline timing, but that this effect cancelled out at the population level, when probed with TMS. Indeed, baseline shifts are usually only observed for a fraction of neurons in single-cells studies ${ }^{25,31}$. At the population level, several studies in humans failed to observe such shifts in the motor cortex, whether using $\mathrm{fMRI}^{29}$, $\mathrm{TMS}^{21}$ or EEG ${ }^{20}$ (although see ${ }^{22}$ ). 
In addition to relying on a broad amplification, hastiness was associated with a local suppression of motor excitability during deliberation, which affected choiceirrelevant representations surrounding the choice-relevant population on the chosen side. This effect is reminiscent of center-surround inhibition mechanisms ${ }^{73-76}$, classically associated with lateral inhibition in the motor cortex. Here, amplification of activity within the neurons of the choice-relevant representation may have resulted in an increased recruitment of inhibitory interneurons connecting them to adjacent populations. Such a mechanism could enhance the signal-to-noise ratio within the representations of the moving effector when context calls for hastiness, allowing for excitatory inputs targeting these representations to better stand out against a quiescent background ${ }^{77-80}$, ultimately reducing the time needed to initiate the action following commitment.

The correlation analyses also support the idea of an enhancement in signal-tonoise ratio within the chosen index representation in the hasty context. The rationale for these analyses was that a high positive correlation between MEPs obtained in the index and in the other finger muscles would indicate the operation of influences exerting a common impact on their representations ${ }^{53,54}$, shaping MEPs in block. As such, shared neural inputs are known to produce correlated fluctuations of neural activity across functionally divergent populations ${ }^{53-58}$. Along these lines, previous studies have found significant correlations between neurons in diverse cortical areas ${ }^{53}$ 55,81-84. Here, we found that excitability changes in the chosen index representation and in the other finger representations decorrelated in the hasty relative to the cautious context, possibly indicating the presence of influences affecting the chosen index representation in a more selective and differentiated way when hastiness was at premium ${ }^{53,55,59}$. In the visual cortex, a similar decorrelation of neural activity has been observed when attention is directed to a stimulus inside a population's receptive field $^{53,54}$. Computational analyses revealed that this attention-driven decorrelation enhances the signal-to-noise ratio of pooled neural signals substantially ${ }^{54}$, a finding in accordance with our current interpretation.

Beyond these context-dependent effects, our data also unveiled an interesting effect of time on motor excitability. In fact, motor excitability displayed a global rise over time during the decision period, which affected all of the representations investigated in the present study. Previous work has shown that activity often rises concomitantly in different choice-relevant representations during sensorimotor decisions (e.g., $12,85,86)$. This finding is usually considered to reflect the unfolding of a competition between neural populations involved in the decision process ${ }^{16,17,87}$. However, our data show that neural activity also builds up over time in choice-irrelevant representations. One potential explanation for this new result is that diffuse modulatory inputs may progressively amplify activity in the sensorimotor system as the urge to act increases during deliberation. In line with this interpretation, several sensorimotor regions - such as the premotor cortex ${ }^{88,89}$, the lateral intraparietal area ${ }^{90,91}$ or the cerebellum ${ }^{15,92,93}$ display time-dependent ramping activities during decision-making. Given its diffuse projections to these structures ${ }^{94}$, the noradrenergic system may represent a potential candidate for this time-dependent modulation. In support of this hypothesis, pupil dilation - a proxy of noradrenergic activity ${ }^{95}$ - also rises as time elapses during deliberation ${ }^{22}$.

Our analysis of voluntary EMG activity suggests that none of these context- and time-dependent changes in motor excitability could be accounted for by alterations in 
movement vigor. Indeed, movement vigor was comparable in the hasty and cautious context as well as for short and long reaction times. At first glance, this finding may appear to contrast with sensorimotor theories of decision-making, postulating that a common decision urgency / movement vigor mechanism would regulate decision and movement speeds $22,70,96-98$. However, recent studies have come to question this unified mechanism view, showing that decision and movement speeds do not necessarily co-vary systematically ${ }^{96,99,100}$. Our findings are therefore in line with these recent observations, and suggest the putative contribution of distinct (yet, overlapping) neural sources to the invigoration of decision-making and action execution processes.

Altogether, our data reveal the concurrent operation of multiple modulatory influences on the motor system during hasty sensorimotor decisions. We found that motor excitability exhibits a global increase as time elapses during the decision process, altering not only choice-relevant representations, but also choice-irrelevant ones that lie far away within the motor system, on both the chosen and the unchosen sides. Beyond this time-dependent effect, the data shows that shifting from a cautious to a hasty context entails a broad amplification of motor excitability, though this amplification was not entirely global as it was limited to the chosen side. Interestingly, on top of this effect, we also identified a local suppression of motor excitability, surrounding the index representation on the chosen side. Hence, a decision policy favoring speed over accuracy appears to rely on overlapping processes producing a broad (but not global) amplification and a surround suppression of motor excitability. The latter effect may help increasing the signal-to-noise ratio of the chosen representation, as supported by the correlation analyses indicating a stronger decoupling of excitability changes between the chosen index representation and the other finger representations in the hasty relative to the cautious context.

\section{ACKNOWLEDGMENTS}

This work was supported by grants from the "Fonds Spéciaux de Recherche" (FSR) of the Université Catholique de Louvain, the Belgian National Funds for Scientific Research (FNRS: MIS F.4512.14) and the "Fondation Médicale Reine Elisabeth" (FMRE). GD was a postdoctoral fellow supported by the FNRS (FNRS: 1B134.18). We thank Sara Lo Presti, Roxanne Weverbergh and Caroline Hermand for their help in the acquisition of the data.

\section{AUTHOR CONTRIBUTIONS}

Conceptualization, G.D., D.T., P.C. and J.D.; Methodology, G.D., D.T., P.C. and J.D.; Investigation, G.D.; Writing - Original Draft, G.D.; Writing - Review \& Editing, G.D., D.T., P.C. and J.D.; Funding Acquisition, G.D. and J.D.; Resources, J.D.; Supervision, J.D.

\section{DECLARATION OF INTERESTS}

The authors declare no competing interests. 


\section{METHODS}

\section{Resource availability}

\section{Lead Contact}

Further information and requests for resources can be directed to and will be fulfilled by the Lead Contact, Gerard Derosiere: gerard.derosiere@uclouvain.be.

\section{Materials Availability}

This study did not generate new unique reagents.

\section{Data and Code Availability}

All datasets and codes generated during this study will be freely available on the Open Science Framework repository upon publication, at: https://osf.io/tbw7h/

\section{Experimental Model and Subject Details}

50 healthy human subjects participated in the study. Among them, 21 received TMS over the finger motor representations (i.e., TMS Finger subjects; 11 women, $24 \pm$ 0.5 years) and 22 received TMS over the leg representations (i.e., TMS Leg Subjects; 14 women, $22.7 \pm 0.3$ years); 7 did not receive TMS and were thus only considered for the behavioral analyses (i.e., No-TMS subjects; 4 women, $21.7 \pm 0.5$ years [mean \pm SE]), Subjects who received TMS answered a medical questionnaire to rule out any potential risk of adverse reactions to brain stimulation. All participants were righthanded according to the Edinburgh Questionnaire and had normal or corrected-tonormal vision. The subjects were financially compensated and provided written informed consent. The protocol was approved by the Ethics Committee of the Université Catholique de Louvain, Brussels, Belgium.

\section{Method details}

\section{Experimental set-up}

Experiments were conducted in a quiet and dimly-lit room. Subjects were seated at a table in front of a 21 -inches cathode ray tube computer screen. The display was gamma-corrected and its refresh rate was set at $100 \mathrm{~Hz}$. The computer screen was positioned at a distance of $70 \mathrm{~cm}$ from the subjects' eyes and was used to display stimuli during the decision-making task. The left and right forearms were rested upon the surface of the table with both hands on a keyboard positioned upside-down. The tip of the left and right index fingers were placed on top of the F12 and F5 keys, respectively (see Figure 1).

Tokens task 
The decision-making task used in the present study is a variant of the tokens task previously exploited to study decisions between reaching movements ${ }^{52}$; it was implemented by means of Labview 8.2 (National Instruments, Austin, TX). The sequence of stimuli in this task is depicted in Figure 1.A. In between trials, a default screen is presented, consisting of three empty blue circles $(4.5 \mathrm{~cm}$ diameter each), placed on a horizontal axis at a distance of $5.25 \mathrm{~cm}$ from each other. The empty circles are displayed on a white background for $2500 \mathrm{~ms}$. Each trial starts with the appearance of fifteen randomly arranged tokens $(0.3 \mathrm{~cm}$ diameter $)$ in the central circle. After a delay of $800 \mathrm{~ms}$, a first token jumps from the center to one of the two lateral circles, starting the deliberation phase. The other tokens then follow, jumping one-by-one every 200 $\mathrm{ms}$, to one of the lateral circles (i.e., 15 token jumps; Jump 1 to Jump ${ }_{15}$ ). In this version of the task, we asked our subjects to choose between left or right index finger keypresses depending on which lateral circle they thought would ultimately receive the majority of the tokens (F12 or F5 key-presses for left or right circle, respectively). They could choose their action and press the related key as soon as they felt sufficiently confident, as long as it was after Jump 1 had occurred and before Jump ${ }_{15}$. After a choice, the tokens kept jumping every $200 \mathrm{~ms}$ until the central circle was empty. At this time, the circle associated with the chosen action turned either green or red depending on whether the choice was correct or incorrect, respectively, providing subjects with a feedback of their performance; the feedback also included a numerical score displayed above the central circle (see below, Reward, penalty and SAT contexts section). In the absence of any key-press before Jump ${ }_{15}$, the central circle became red and a "Time Out" message appeared on top of the screen. The feedback screen lasted for $500 \mathrm{~ms}$ and then disappeared at the same time as the tokens did (the circles remained on the screen), denoting the end of the trial. Each trial lasted $6600 \mathrm{~ms}$.

For each trial, we defined the "success probability" $p_{i}(t)$ associated with choosing each action (i.e., left or right key-press) at each moment in time. If at a moment in time, the left $(L)$ circle contains $N_{L}$ tokens, the right $(R)$ one contains $N_{R}$ tokens, and $N_{C}$ tokens remain in the central $(\mathrm{C})$ circle, then the probability that the left response is ultimately the correct one (i.e., the success probability of guessing left) is as follows:

$$
p\left(L \mid N_{L}, N_{R}, N_{C}\right)=\frac{N_{C} !}{2^{N_{C}}} \sum_{k=0}^{\min \left(N_{C}, 7-N_{R}\right)} \frac{1}{k !\left(N_{C}-k\right) !}
$$

Calculating this quantity for the 15 token jumps allowed us to construct the temporal profile of success probability $p_{i}(t)$ for each trial. As far as the subjects knew, the individual token movements and the correct choice were completely random. However, we interspersed distinct trial types within the full sequence of trials. First, in $60 \%$ of trials, the $p_{i}(t)$ remained between 0.5 and 0.66 up to Jump $10-i . e$., the initial token jumps were balanced between the lateral circles, keeping the $p_{i}(t)$ close to 0.5 until late in these "ambiguous" trials. Second, in $15 \%$ of trials, the $p_{i}(t)$ was above 0.7 after Jump 3 and above 0.8 after Jump - i.e., the initial jumps consistently favored the correct choice in these "obvious" trials. Then, in another $15 \%$ of trials, the $p_{i}(t)$ was below 0.4 after Jump $_{3}-$ i.e., the initial jumps favored the incorrect choice and the following ones favored the correct choice in these "misleading" trials. The remaining $10 \%$ of trials were fully random (i.e., putatively involving ambiguous, obvious and 
misleading trials, as well as other trials with different $\left.p_{i}(t)\right)$. Critically, the ambiguous trials were more frequent $(60 \%)$ than the other trial types $(30 \%)$ because they represented our main condition of interest and their high prevalence allowed us to obtain enough probes of motor excitability during the course of deliberation in this specific setting (see below, TMS intensity and timings section).

\section{Reward, penalty and SAT contexts}

As mentioned above, subjects received a feedback score at the end of each trial depending on whether they had chosen the correct or the incorrect circle. Correct choices led to positive scores (i.e., a reward) while incorrect choices led to negative scores (i.e., a penalty). Subjects knew that the sum of these scores would turn into a monetary gain at the end of the experiment.

The reward provided for correct choices was equal to the number of tokens remaining in the central circle at the time of the key-press (in $€$ cents); hence, it gradually decreased as time elapsed in each trial (see Figure 1.B). For instance, a correct choice led to a reward of +10 cents when the response was provided between Jump$_{5}$ and Jump 6 (10 tokens remaining in the central circle). However, it only led to a reward of +5 cents when the response was provided between Jump 10 and Jump 11 (5 tokens remaining in the central circle). The fact that the potential reward progressively dropped produced a speed/accuracy trade-off, as subjects wanted to decide fast enough to get a large reward but also slow enough to choose the correct target and avoid the penalty. This SAT has been proposed to be set by a context-dependent urgency signal that grows over time during deliberation, as evidenced from the urgency functions obtained in such tasks ${ }^{60,61}$ (see also Figure 2).

By contrast, the penalty provided for incorrect choices was constant throughout deliberation. Importantly though, it differed in two block types, producing two SAT contexts. In the first block type, incorrect choices were severely sanctioned as the penalty there was of -14 cents, emphasizing the need for cautiousness (cautious context). Conversely, the cost of making an incorrect choice was low in the second block type as the penalty was only of -4 cents, encouraging subjects to make hasty decisions in order to get high reward scores (hasty context). Hence, by manipulating the monetary cost associated with incorrect choices, we aimed at instigating distinct levels of urgency in two separate contexts (high and low urgency in hasty and cautious contexts, respectively), as confirmed by the analyses run on the behavioral data (please see Figure 2 and Figure S2).

Finally, not providing a response before Jump 15 (i.e., no-response trials) also led to a penalty, which was of -4 cents both in the hasty and in the cautious contexts. Hence, in the hasty context, providing an incorrect response or not responding led to the same penalty (i.e., -4 cents), further increasing the urge to respond before the end of the trial. Conversely, in the cautious context, the potential penalty for making an incorrect choice was much higher than that obtained for an absence of response (i.e., -14 vs -4 cents, respectively), further increasing subjects' cautiousness in this context.

Time course of the sessions 
The study included two experimental sessions conducted at a 24-h interval. In each session, subjects realized the task in one SAT context; we thus refer to those as hasty and cautious sessions. The order of the sessions was counterbalanced across participants. Further, in order to prevent our data from being confounded by a potential difference in chronobiological states, the subjects were always tested at the same time of the day ${ }^{101-103}$.

The two sessions involved the same sequence of blocks. Each session started with two short blocks of a simple RT (SRT) task. This SRT task involves the same display as in the tokens task described above ${ }^{60,61}$. However, here, the 15 tokens remain only $50 \mathrm{~ms}$ in the central circle, after which they jump altogether simultaneously into one of the two lateral circles (always the same one in a given block). Subjects were instructed to respond to this "GO signal" by pressing the appropriate key with the corresponding index finger (i.e., F12 and F5 for right and left circles, respectively). Because the circle was known in advance of the block, the task did not require any choice to be made; it was exploited to determine the subject's median SRT for left and right index finger key-presses ${ }^{52}$.

Then, subjects performed training blocks to become acquainted with the tokens task. In a first training block (10 trials, only run on the first session), we ran a version of the tokens task in which the feedback was simplified; the lateral circle turned green or red, depending on whether subjects had chosen the correct or the incorrect action but no reward or penalty was provided here. Two training blocks were then realized with the full version of the task (involving rewards and penalties), one for each SAT context (20 trials each). Subjects performed a last training block (20 trials), which involved the SAT context that they would be performing next during the whole session. This last block also involved TMS, which was either applied to the finger motor representation (TMS Finger subjects) or the leg representation (TMSLeg subjects), to prepare participants to the pulse sensation during the task. No-TMS subjects realized the last training block without TMS.

The actual experiment involved 8 blocks of 40 trials (regardless of choice outcome) in which subjects performed the tokens task with online TMS (320 trials per session). Each block lasted about 4"30 minutes (40 trials of $6600 \mathrm{~ms}$ each) and a break of 2 to 5 minutes was provided between each block. The maximal duration of a session was 120 minutes.

\section{Transcranial magnetic stimulation (TMS) over finger representations}

In TMS Finger subjects $(n=21)$, pulses were delivered using a double-coil protocol whereby both $\mathrm{M} 1$ areas are stimulated at a near-simultaneous time (1-ms delay; right M1 pulse first), eliciting MEPs in finger muscles of both hands that are statistically equivalent to those obtained using classic single-coil TMS ${ }^{44,104-106}$ (see Figure 1.C and Figure S1.A and B). Both pulses were delivered through small figure-of-eight coils (wing internal diameter of $35 \mathrm{~mm}$ ), which were connected to monophasic Magstim stimulators (one Magstim 200 and one Magstim Bistim²; Magstim, Whitland, Dyffed, UK; the side of the stimulators was counterbalanced across participants). We placed the two coils tangentially on the left and right side of the scalp with the handles oriented 
towards the back of the head and laterally at a $45^{\circ}$ angle away from the midline (see Figure 1.C).

Our objective in this group of subjects was to map the spatiotemporal changes in motor excitability occurring in populations of corticospinal cells projecting to finger muscles (i.e., occurring in finger motor representations) during the index finger choices in the tokens task, in the hasty and cautious contexts. To do so, we examined MEPs in three different muscles, namely, the first dorsal interosseous (FDI; index finger abductor), the abductor pollicis brevis (APB; thumb abductor) and the abductor digiti minimi (ADM; pinky abductor). The FDI being prime mover in the task, its MEPs allowed us to observe excitability changes associated with a choice-relevant motor representation. As for the APB and ADM, these muscles being not required in the task, their MEPs allowed us to assess excitability changes associated with choice-irrelevant representations that lie close by the prime mover representation in the motor system (i.e., in terms of somatotopy). Importantly, MEPs in these three muscles were obtained by stimulating a single spot. This hotspot was found for each M1 at the beginning of every single session in each subject; it corresponded to the hotspot of the ADM ${ }^{107}$, which usually provides the most consistent MEPs when these three muscles are considered together (see Figure S1.A). Further, eliciting concurrent MEPs in both hands allowed us to capture excitability changes on the two sides of the motor system at once (in each trial), thus concerning finger representations that are both on the side of the chosen index and on the side of the unchosen finger (e.g., right and left MEPs, respectively, preceding a right index finger choice). Hence, each double-coil stimulation allowed us to obtain six MEPs, reflecting the excitability of six different finger representations playing distinct roles in the task (i.e., index, thumb and pinky representations on the chosen and unchosen sides). The two M1 sites were marked on an electroencephalography cap fitted on the subject's head to provide a reference point throughout the experimental session ${ }^{13,14,108}$.

\section{TMS over leg representations}

In TMS Leg subjects $(n=22)$, TMS was applied over the leg representation of the left M1, using a batwing coil (D-B80 Magpro coil) connected to a Magpro X100 Stimulator (Magventure, Farum, Denmark). A batwing coil had to be used here because leg muscles are represented deep into the interhemispheric fissure and are difficult to target using figure-of-eight coils, which mainly activate superficial neural layers ${ }^{109}$. Further, we decided to use biphasic pulses because they are known to activate deep neurons more efficiently than monophasic pulses ${ }^{110,111}$.

Our objective in this group of subjects was to map the changes in motor excitability occurring for corticospinal cells projecting to leg muscles (i.e., in leg representations) during the index finger choices of the tokens task, in the hasty and cautious contexts. To do so, we examined MEPs in three different muscles of the right leg, including the tibialis anterior (TA), as well as the lateral and medial heads of the gastrocnemius (LG and MG, respectively). These muscles being not required in the task, their MEPs allowed us to assess changes associated with choice-irrelevant representations that lie far from the prime mover representation in the motor system in terms of somatotopy. Similar as for the finger muscles, MEPs in all three leg muscles were obtained by stimulating a single hotspot. To do so, the coil was initially placed tangentially on the 
vertex of the scalp with the handle oriented towards the back of the head and parallel to the midline. Then, we turned the handle incrementally following an anticlockwise direction in order to orient the magnetic field towards the leg representation in the left M1 and to obtain maximal MEP amplitudes in the right TA muscle. Although of smaller amplitude, TMS at this location evoked consistent MEPs in the LG and MG muscles too (see Figure S1.B), allowing us to broaden our observations to two other choiceirrelevant leg representations (see Figure S1.B and D as well as Figure S4). Here, MEPs were only obtained in right leg muscles (they were never elicited in the left leg). However, because the tokens task requires deciding between right and left index finger choices, right leg MEPs could be classified according to whether they fell on the same side as the chosen index (in right hand trials) or on the side of the unchosen index (in left hand trials). Hence, this design allowed us to capture excitability changes associated with leg representations of both the chosen and unchosen index sides. Similar as for the TMS Finger subjects, the hotspot was marked on an electroencephalography cap fitted on the subject's head, providing a reference point throughout the session.

\section{TMS intensity and timings}

The intensity of stimulation was set in the same way in $\mathrm{TMS}_{\text {Finger }}$ and TMS $\mathrm{Teg}$ subjects. Once the hotspot was located, we first determined the individual resting motor threshold ( $\mathrm{rMT})$, defined as the minimal intensity required to evoke MEPs of $50 \mu \mathrm{V}$ peak-to-peak on 5 out of 10 consecutive trials in the contralateral ADM or TA muscle. The ADM was used as reference in the TMS Finger subjects because it is usually associated with a slightly higher rMT than the FDI and APB; so in this way we obtained MEPs that are big enough in all muscles. As for the TMS Leg subjects, setting the rMT based on the TA also allowed us to obtain reliable MEPs in the two other muscles.

The rMT was similar in the hasty and the cautious sessions in TMS Finger subjects, both for the right hemisphere $(45.85 \pm 2.12 \%$ and $46.19 \pm 1.94 \%$ of the maximum stimulator output [MSO], respectively) and for the left hemisphere (45.57 $\pm 1.96 \%$ and $46.23 \pm 2.15 \% \mathrm{MSO}$, respectively). This was also the case for the rMT of left hemisphere in the TMS Leg subjects $(51.27 \pm 1.73 \%$ and $51.68 \pm 1.71 \%$ MSO in the hasty and the cautious sessions, respectively). In each session, TMS pulses were then applied at $120 \%$ of the rMT during the whole experiment ${ }^{112}$.

TMS was applied both outside the blocks (i.e., at rest) and at specific timings during the blocks. MEPs elicited outside of the blocks allowed us to probe the resting-state level of motor excitability in both sessions. We recorded 20 to $25 \mathrm{MEPs}$, depending on their variability, before and after the 8 blocks of trials. Importantly, the amplitudes of these resting-state MEPs were comparable in the hasty and the cautious sessions,

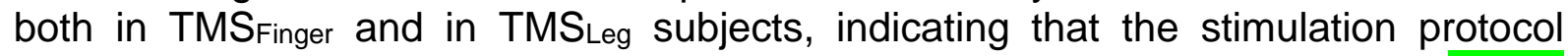
guaranteed reproducible measurements across experimental sessions (see Figure S1.B and D for details).

When applied during the blocks, TMS could occur at one of four different timings (see Figure 1.C). First, it could occur when the circles were empty (i.e., $500 \mathrm{~ms}$ before the appearance of the tokens in the central circle), allowing us to measure the baseline level of motor excitability while subjects were at rest but engaged in the task. Moreover, TMS could occur at one of three different token jumps: Jump 1 , Jump 4 or Jump (i.e., 
corresponding to 0,600 and $1200 \mathrm{~ms}$ from deliberation onset). The MEPs recorded at these timings served to probe the changes in motor excitability during the deliberation process.

MEPs were elicited in about $90 \%$ of the total number of trials in both contexts ( $\mathrm{n}$ $=291 / 320$ ). Hence, about $10 \%$ of trials did not involve TMS (about 4 trials per block), preventing subjects from anticipating the stimulation. Further, the percentage of stimulated trials was the same across trial types (i.e., $90 \%$ of ambiguous, obvious, misleading and random trials), such that subjects could not associate a particular trial type with TMS. However, the MEPs elicited in obvious and misleading trials $(n=94$ in total, including all TMS timings) were not exploited in our analyses, as those usually involve RTs that occur before Jump 4 and Jump $7^{88}$. As such, while designing the sequence of trials, we anticipated to focus on ambiguous trials for the analysis of the MEPs elicited at Jump 1 , Jump 4 and Jump J $_{7}$ as these trials are associated with longer RTs. Further, ambiguous trials are characterized by a distribution of tokens that remains balanced across the two lateral circles until Jump 10 . Thus, MEP changes occurring before this time in ambiguous trials (i.e., from Jump 1 to Jump7) could not be related to any fluctuation in sensory evidence, which is known to influence motor activity ${ }^{113-115}$. In total, for each session (i.e., in each context), 170 MEPs were elicited in ambiguous trials, among which 58 were obtained at Jump 1,56 at Jump 4 and 56 at Jump7. Baseline MEPs were elicited in the random trials $(n=27)$.

\section{Electromyography (EMG) data collection}

Surface EMG electrodes (Medicotest, USA) were placed on the investigated muscles (i.e., right and left FDI, APB and ADM in TMS Finger subjects and right TA, LG and MG in TMS Leg subjects), allowing us to record the MEPs elicited in these muscles. The EMG signals recorded in TMS Finger subjects were also exploited to quantify movement vigor (see below). The ground electrode was placed over the right ulnar styloid process in the TMS Finger subjects and over the right patella in the TMS subjects. The signals were recorded for $4000 \mathrm{~ms}$ on each trial, starting $500 \mathrm{~ms}$ before the first TMS timing (i.e., before baseline) and ending $1000 \mathrm{~ms}$ after the last TMS timing (i.e., after Jump7). The EMG signals were amplified, band-pass filtered $(10-500 \mathrm{~Hz})$ and notch filtered $(50 \mathrm{~Hz})$ on-line (NeuroLog, Digitimer, UK), and digitized at $2000 \mathrm{~Hz}$ for off-line analysis.

\section{Quantification and statistical analysis}

Behavioral data were collected by means of LabView 8.2 (National Instruments, Austin, TX), stored in a database (Microsoft SQL Server 2005, Redmond, WA), and analyzed with custom Matlab (MathWorks, Natick, MA) and R scripts (R Core Team, 2020). EMG data were collected using Signal 6.04 (Cambridge Electronic Design, Cambridge, UK) and analyzed with custom Signal and R scripts. Statistical analyses were performed using custom R scripts and Statistica 7.0 (Statsoft, Oklahoma, UnitedStates).

\section{Decision behavior quantification}


For each subject and each SAT context, we computed the median decision time (DT; all trial types pooled together) and decision accuracy (i.e., percentage of correct choices over total number of choices made). To estimate the DT in each trial, we first calculated the reaction time (RT) during the tokens task by computing the difference between the time at which the subject pressed the key and the time of Jump 1 . We then subtracted from the single-trial RTs the median SRT for each subject (i.e. difference between key-press and the tokens' jump in the SRT task). This procedure allowed us to remove from the individual RT obtained in the tokens task, the sum of the delays attributable to sensory processing of the stimulus display as well as to response initiation and muscle contraction, providing us the DT52.

The tokens task also allowed us to estimate the amount of evidence based on which subjects made their action choices in each SAT context. To do so, we first computed a first-order approximation of the real probability function after each jump (see equation 1), called the sum of log-likelihood ratios (SumLogLR) ${ }^{52,98}$ :

$$
\operatorname{SumLogLR}(n)=\sum_{k=1}^{n} \log \frac{p\left(e_{k} \mid C\right)}{p\left(e_{k} \mid U\right)}
$$

In this equation, $p\left(e_{k} / C\right.$ ) is the likelihood of a token event $e_{k}$ (a token favoring either the chosen or the unchosen action) during trials in which the chosen action $C$ is correct and $p\left(e_{k} \mid U\right)$ is the likelihood of $e_{k}$ during trials in which the unchosen action $U$ is correct. The SumLogLR is proportional to the difference between the number of tokens that favored each of the two possible choices (i.e., that moved towards each lateral circle) at any given time. Hence, the lower the amount of sensory evidence in favor of the chosen action, the lower the SumLogLR. To characterize the decision policy of the subjects in each SAT context, we determined the level of sensory evidence at the time of commitment (i.e., at DT). To do so, we grouped the trials into 10 consecutive percentile bins of DT (DTBin1-10), and then calculated the average SumLogLR for each bin in each subject.

We exploited the obtained SumLogLR at DT values to estimate urgency functions. As such, models of decision-making that incorporate an urgency signal, posit that choices result from the combination of signals that reflect the available sensory evidence and the level of urgency that grows over time (e.g., $\left.{ }^{62,63}\right)$. For instance, in a minimal implementation of the urgency-gating model ${ }^{52,98}$, evidence is multiplied by a linearly increasing urgency signal and then compared to a fixed decision threshold. The result can be expressed as follows:

$$
y_{i}=\left(N_{i}-N_{j \neq i}\right) \cdot[a t+b]^{+}<T
$$

Where $y_{i}$ is the "neural activity" for action choices to lateral circle $i, N_{i}$ is the number of tokens in lateral circle $i, t$ is the number of seconds elapsed since the start of the trial, $a$ and $b$ are the slope and y-intercept of the urgency signal, and [ ]+ denotes halfwave rectification (which sets all negative values to zero). When $y_{i}$ for any action crosses the threshold $T$, that action is chosen. 
A direct implication of such urgency-based models is that decisions made with low levels of sensory evidence should be associated with high levels of urgency and vice versa. That is, one core assumption is that a high urgency should push one to commit to a choice even if evidence for that choice is weak. Hence, considering a model in which evidence is multiplied by an urgency signal, we estimated urgency values based on the SumLogLR at DT obtained in each subject, at each bin, and in each SAT context, as follows:

$$
U_{(s, t, c)}=\frac{T}{S L R_{(s, t, c)}}
$$

In the above, $s$ is the subject number, $t$ is the DT bin, $c$ is the SAT context, $S L R$ is the SumLogLR at DT, $T$ is a constant representing a fixed threshold (which we fixed to 1 ), and $U$ is the estimated urgency value. We then fitted a linear regression model over the obtained urgency values, and extracted the intercept and the slope of the functions for each subject and both contexts.

\section{Movement vigor quantification}

We also examined the vigor with which the subjects pressed the response key in the hasty and cautious contexts. To do so, we exploited the EMG signals recorded in the finger muscles in TMS Finger subjects (i.e., in left and right index, thumb and pinky muscles) and considered the magnitude of EMG burst preceding the key-press as a proxy of movement vigor 22,70 .

First, the signals were segmented into epochs extending from -300 to $0 \mathrm{~ms}$ with respect to the key-press (i.e., 600 data points). Trials in which a TMS pulse occurred between -400 and $0 \mathrm{~ms}$ were discarded from the analysis, preventing contamination of the segmented signals from TMS artifacts and MEPs. For each epoch, we then removed any putative signal offset by subtracting the average signal amplitude in the first 50 ms from every data point of the epoch. The signals were subsequently rectified by taking the absolute value of each data point.

In a following processing step, we classified the epochs according to the individual's RT in the trial, allowing us to test for any impact of elapsed time on movement vigor (in addition to the impact of context). Epochs were categorized depending on whether they were associated with a short or a long RT using a mediansplit approach (RTshort or RTLong, respectively). Further, given the expected betweencontext difference in RTs and its potential effect on movement vigor ${ }^{18,98}$, we adopted a RT-matching procedure to homogenize $R T_{\text {short }}$ and $R T_{\text {Long }}$ distributions across contexts $^{20}$. The procedure consisted in discretizing each subject's RT Short and RTLong distributions into bins of $200 \mathrm{~ms}$ width and, for each bin, randomly selecting a matched number of trials from the context condition that had the greatest trial count in that bin. One subject had to be discarded from the analysis at this step because the overlap between her/his RT distributions across contexts was too small, leaving less than 6 trials for some conditions after the matching procedure. The remaining 20 subjects presented an average of $62 \pm 2$ trials per RT Length and context (range: [50 - 73 trials]). Following this step, the trials included in the analysis involved homogenous RTshort and RT Long across the hasty and cautious contexts, as depicted in Figure S6 (RT Short: 1417 
$\pm 46 \mathrm{~ms}$ and $1422 \pm 46 \mathrm{~ms}$, respectively; RT Long: $2030 \pm 20 \mathrm{~ms}$ and $2034 \pm 19 \mathrm{~ms}$, respectively).

We then computed the median value of each data point across the epochs for each condition of interest, providing us with 24 signals per subject: that is, one signal was obtained for each muscle (index, thumb, pinky muscles), each hand (chosen, unchosen), each RTLength (RT Short, RTLong) and each SAT context (ContextHasty, Contextcautious). These signals were baseline-corrected (i.e., baseline-subtraction; reference window: -300 to $-200 \mathrm{~ms}$ ) and low-pass filtered (butterworth filter; order: 1, cut-off frequency: $5 \mathrm{~Hz}$ ). Three variables were finally extracted to quantify movement vigor in each condition in the chosen hand: the maximal peak amplitude and the timeto-peak amplitude. The latest variable was estimated by computing the difference between the maximal peak timing and the onset of voluntary contraction (estimated using a threshold of 3 standard deviation [SD] above the average signal amplitude in a window extending from -300 to $-200 \mathrm{~ms}$ ).

\section{Motor excitability quantification}

Motor excitability was quantified based on the absolute peak-to-peak amplitude of MEPs (in $\mu \mathrm{V}$ ) in each target muscle of the TMS Finger and TMS Leg $_{\text {subjects. As mentioned }}$ above, MEPs elicited at Jump ${ }_{1}$, Jump 4 and Jump 7 were only considered in ambiguous trials. Moreover, we only included trials in which the RT was comprised between 1350 and $2800 \mathrm{~ms}$ (i.e., at least $150 \mathrm{~ms}$ after Jump 7 and up to Jump 15 ; see Figure S5). Hence, even in trials with TMS at the latest time point (Jump7), the selected trials involved MEPs that fell relatively far from movement onset (at least $150 \mathrm{~ms}$ before the key-press), allowing us to capture motor excitability changes that are specific to deliberation and not movement execution. No-response trials were excluded from the analysis.

In order to prevent contamination of the measurements from background muscular activity, trials in which the root mean square of the EMG signal exceeded 3 SD above the mean before stimulation (i.e., -250 to $-50 \mathrm{~ms}$ from the pulse) were discarded from the analyses (rejection rate: $8.48 \pm 0.43 \%$ in TMSFinger Subjects and $0.99 \pm 0.05 \%$ in TMS Leg subjects). MEPs with an amplitude exceeding 3 SD around the mean were excluded too (rejection rate: $3.70 \pm 0.42 \%$ in TMS Finger subjects and $2.37 \pm 0.24 \%$ in TMS Leg subjects).

Following this cleaning procedure in the TMS Finger subjects, we had $39 \pm 0.3$ and $38.8 \pm 0.2$ trials left with TMS falling outside of the blocks (i.e., at rest) in the hasty and cautious sessions, respectively. For the analysis of motor excitability in these restingstate trials, we first computed separate medians of MEP amplitude for each hand, and this, for each finger representation, each SAT context and each subject. We then further pooled the MEP data from the left and right hands to obtain a single restingstate motor excitability value for each finger representation, context and subject. In the TMS Leg subjects, we were left with $46.2 \pm 0.4$ and $45.4 \pm 0.3$ resting-state trials in the two corresponding sessions. Here, MEPs were only elicited in the right leg and separate medians were computed for each motor representation (i.e., of the TA, MG and LG muscles), each context and each subject. 
Trials in which MEPs occurred at Jump 1 , Jump 4 and Jump 7 were further processed using a RT-matching procedure, allowing us to homogenize RT distributions across contexts for each TMS timing separately (see Figure S3). Following this step, 2 TMS Finger and 6 TMS Leg subjects had to be discarded from the analysis because their datasets fell to less than 8 trials on average across TMS timings (the behavioral data and the baseline and resting-state MEP data of these subjects were conserved in the respective analyses). The datasets of the remaining $19 \mathrm{TMS}_{\text {Finger }}$ and $16 \mathrm{TMS}$ Leg subjects comprised an average of $28 \pm 2$ and $15 \pm 1$ trials, respectively, across TMS timings and SAT contexts (range: [10 - 39 trials] and [8 - 27 trials]). The included trials involved comparable RTs in hasty and cautious contexts, both in TMS Finger subjects

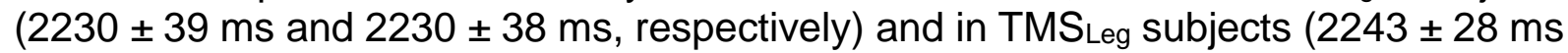
and $2249 \pm 26 \mathrm{~ms}$, respectively).

Preliminary analyses showed that, if performed multiple times, the trial selection of the matching procedure could produce subtle variations in MEP amplitudes when trials were then pooled across conditions (e.g., across TMS timings, contexts, etc.), depending on which trials were eventually included in the analysis. Hence, to avoid any effect of the trial selection on the results, the procedure was repeated 100 times, the median MEP amplitude was first calculated for each condition and for every iteration and we then calculated the median MEP amplitude across iterations (see ${ }^{20}$, for a similar procedure). Following this step in TMS Finger subjects, one MEP amplitude was obtained for 72 conditions, namely for each TMS timing (Jump1, Jump4, Jump7), each context (hasty, cautious), each of the six motor representations (left and right FDI, APB and ADM), when these representations were classified as part of the chosen or the unchosen side of the motor system. In TMS Leg subjects, one MEP amplitude was obtained for each TMS timing (Jump 1 , Jump4, Jump 7 ), each context (hasty, cautious), each of the three motor representations (right TA, LG and MG), and each side (chosen, unchosen). Besides, baseline MEP amplitudes were not subjected to the RT-matching procedure and were directly pooled together for each context and each representation, independently of the side that ended up being chosen.

Once the median MEP amplitudes were obtained (in $\mu \mathrm{V}$ ), we normalized them (in $\%)$. That is, MEPs obtained at baseline were expressed in percentage of resting-state amplitudes ${ }^{65,116}$, providing us with a normalized measure of baseline excitability for each motor representation and each context. Further, amplitudes obtained at Jump , $_{1}$ Jump $_{4}$ and Jump 7 were expressed in percentage of baseline amplitudes ${ }^{78,117}$, providing a normalized measure of excitability for each motor representation on the side of the chosen and unchosen index fingers, in each context. Notably, in TMS Finger subjects, we first normalized separately MEPs associated with left and right finger representations and then pooled the obtained values together according to whether they fell on the side of the chosen or the unchosen index finger.

Ultimately, we computed spatiotemporal maps to provide an integrative view of motor excitability changes occurring during the course of deliberation in each context. To this aim, we considered the MEPs obtained for the index (FDI), thumb (APB), pinky (ADM) and leg (TA, LG, MG pooled together) representations on the side of both the chosen and unchosen index fingers (i.e., 8 representations). For each representation in each context, we averaged excitability across participants and then performed a linear interpolation to estimate excitability changes between each timing (100 data points between each timing), providing us with a temporally continuous trace. For each context, the 8 traces were then spatially arranged according to M1 somatotopy: that is, 
traces of the thumb, index, pinky and leg representations on the chosen side (i.e., latero-medial arrangement) were followed by traces of the unchosen leg, pinky, index and thumb representations (i.e., medio-lateral arrangement). Here again, a linear interpolation was performed to estimate excitability changes between each representation (100 data points), providing us with a spatially continuous trace at each time point. Two spatiotemporal maps were thus obtained (one for each context) and a between-context difference map was finally computed (i.e., hasty minus cautious context).

\section{Single-trial correlation of motor excitability between the chosen index and other finger representations}

In the TMS Finger subjects, the use of double-coil TMS allowed us to obtain MEPs from six finger muscles at once in each trial. Hence, besides considering the amplitude of MEPs within each of these muscles separately, we could also assess the degree to which MEPs in these different muscles varied in concert from one trial to another, providing us with a measure of their relationship in terms of changes in motor excitability. Here, we focused on the link between the chosen index finger and each of the five other finger representations. To do so, we exploited an approach inspired by seed-based correlation analyses (SCAs), which are usually applied on neuroimaging data to quantify correlations between activity changes in a specific region of interest (i.e., the seed) and other brain regions (e.g., ${ }^{118-120}$ ). For the purpose of this study, we defined the representation of the chosen index finger as our seed and quantified the relationship between this key representation and each of the five other finger representations (i.e., thumb and pinky on the same (chosen) side, as well as index, thumb and pinky on the unchosen side). The rationale here was that a high positive correlation between the index and the other finger muscles would indicate the operation of influences exerting a broad, common impact on their motor representations ${ }^{53,54}$, shaping MEPs in block. In contrast, a low or even a negative correlation would indicate the presence of influences affecting the chosen index representation in a more selective and differentiated way. We were interested in comparing the strength of the bond linking the chosen index to the other fingers between both contexts.

To this aim, we exploited the single-trial MEPs obtained at Jump 7 following the 100 iterations of the RT-matching procedure described above. We first normalized singletrial MEPs as a percentage of the average baseline amplitude for each finger representation, each timing and each context and then computed Pearson's correlations between the five pairs of muscles for each timing and each context. We considered the trials of every subjects altogether ( $\mathrm{N}_{\text {subjects }}=19$ ), providing us with a large pool of data points $\left(\mathrm{N}_{\text {Trials }}=479\right)$. Importantly, given the RT-matching procedure, the number of trials for a given subject was equal in each context, such that each one had the same weight in each correlation. To normalize distribution of the single-trial data, we applied a square root transformation on each data point (the findings presented in Figure 6 still hold without this transformation, as presented in Figure S6). Further, we adopted a permutation-based analysis to estimate statistical significance of each correlation (Nermutations $=1000)^{67}$. The whole procedure was repeated 100 times (i.e., corresponding to the 100 pools of data points obtained following the RTmatching procedure), providing us with 100 R-values and 100 permutation-based p- 
values. We finally calculated the median of these $\mathrm{R}$ - and $\mathrm{p}$-values across iterations as estimate values of the correlations. Given that 10 correlations were performed (i.e., 5 representation pairs in both contexts), the significance threshold was set at .005 after Bonferroni correction. The impact of the context on the correlation was tested by comparing R-values using dedicated statistical tests (see Statistical analysis section, below).

\section{Statistical analysis}

No-TMS, TMS Finger and TMS Leg subjects all exhibited strongly similar decision behavior (presented in detail in Figure S2). Hence, the behavioral data of the 50 subjects were considered altogether in a single statistical analysis (Figure 2). First, a permutation-based Pearson's correlation was realized to test any significant relationship between DTs and decision accuracy in each context (Nermutations $=1000)$. The DT, decision accuracy, urgency slope and intercept data were then compared across contexts using two-tailed Student's t-tests for paired-samples. For each context, the slope of the urgency function was further compared against 0 using a two-tailed ttest. Effect sizes were estimated for each t-test by calculating Cohen's d values. In accordance with conventional interpretation of Cohen's $d$, a value of 0.2 is interpreted as indicating a small effect size, a value of 0.4 a medium effect size, and a value of 0.8 or more as a large effect size ${ }^{121}$.

Most of the ensuing statistical comparisons involved repeated-measures analyses of variance (rmANOVAs). When performing rmANOVAs, Maunchley's tests were exploited systematically to check for data sphericity and Greenhouse-Geisser (GG) corrections were used to correct for any deviation from sphericity. Post-hoc comparisons were conducted using the Tukey's Honestly Significant Difference (HSD) procedure. Effect sizes were estimated for each main effect and interaction by calculating partial eta squared $\left(n^{2}\right)$. In accordance with conventional interpretation partial $\eta^{2}$, a value of 0.01 is interpreted as indicating a small effect size, a value of 0.06 a medium effect size and a value of 0.14 or more as a large effect size ${ }^{122}$.

The effect of elapsed time and context on movement vigor was tested using threeway rmANOVAs on the maximal peak amplitude and the time-to-peak amplitude data with MUSCLE (index, thumb, pinky muscles), RTLENGTH (RTshort, RT Long) and CONTEXT (hasty, cautious) as within-subject factors.

Normalized excitability data obtained at baseline were analyzed using two-way rmANOVAs with REPRESENTATION (index, thumb, pinky in TMS Finger subjects and tibialis, lateral and medial gastrocniemius in TMS Leg subjects) and CONTEXT (hasty, cautious) as within-subject factors. In addition, excitability data measured during deliberation on the side of the chosen and unchosen index fingers were analyzed using two separate three-way rmANOVAs with TIMING (Jump 1 , Jump4, Jump 7 ), REPRESENTATION and CONTEXT as within-subject factors.

When a rmANOVA pointed to a lack of significant effect, a Bayes factor analysis was performed to quantify statistically the level of evidence for a lack of effect. Bayes factors provided us with a ratio of the likelihood probability of the null hypothesis (i.e., $\mathrm{HO}$ : the probability that data do not exhibit an effect of factor tested) over the alternative hypothesis (i.e., $\mathrm{H} 1$ : the probability that data exhibit the effect; Morey and Rouder 
2011). A Bayes factor value of 1 would reflect an equal probability that $\mathrm{H} 0$ and $\mathrm{H} 1$ are correct, whereas a Bayes factor value higher than 1 would reflect a higher probability that $\mathrm{HO}$ is correct. In accordance with conventional interpretation of Bayes factor values $^{123}$, a Bayes factor ranging between 1 and 3 is interpreted as indicating anecdotal evidence in favor of $\mathrm{H} 0$, a value between 3 and 10 as indicating substantial evidence for $\mathrm{HO}$, a value between 10 and 100 a strong evidence for $\mathrm{H} 0$, and a value above 100 a decisive evidence for $\mathrm{H} 0$.

Finally, we tested the effect of context on the single-trial correlations. As such, permutation-based $p$-values allowed us to identify changes in the significance of the correlations between contexts. However, in order to quantify such changes more directly, we compared the strength of the correlation between contexts using Fisher's $Z$ procedure ${ }^{68}$, which determines the significance of a difference between R-values. Given that this procedure was repeated 5 times, the significance threshold was Bonferroni-corrected at 01.

\section{Key resources table}

\begin{tabular}{lll}
\hline $\begin{array}{l}\text { REAGENT } \\
\text { RESOURCE }\end{array}$ & / SOURCE & IDENTIFIER \\
\hline Software and Algorithms & \\
\hline MATLAB 2008 & Mathworks & $\underline{\underline{h t p s: / / w w w . m a t h w o r k s . c o m / p r o d u c t / m a t l a b . h ~}}$ \\
\hline LabVIEW 8.2 & $\begin{array}{l}\text { National } \\
\text { Instruments }\end{array}$ & $\underline{\underline{\text { http://www.ni.com/fr-fr/shop/labview.html }}}$ \\
\hline R & R Core Team & $\underline{\text { https://www.R-project.org/ }}$ \\
\hline Signal & $\begin{array}{l}\text { Cambridge } \\
\text { Electronic } \\
\text { Design }\end{array}$ & $\underline{\text { https://ced.co.uk/en/ }}$ \\
\hline D360 Software & Digitimer & $\underline{\text { https://www.digitimer.com/product/life- }}$ \\
& $\underline{\text { science-research/amplifiers/d360-8-channel- }}$ \\
\hline Other & $\underline{\text { patient-amplifier/ }}$ \\
\hline $\begin{array}{l}\text { Magstim 2002 \& } \\
\text { BiStim }\end{array}$ & Magstim & $\underline{\text { https://www.magstim.com/ }}$ \\
\hline D360 Amplifier & Digitimer & $\underline{\text { https://www.digitimer.com/product/life- }}$ \\
& & $\underline{\text { science-research/amplifiers/d360-8-channel- }}$ \\
\hline Datient-amplifier/
\end{tabular}




\section{REFERENCES}

1. Heitz, R.P. (2014). The speed-accuracy tradeoff: History, physiology, methodology, and behavior. Front. Neurosci. 8, 150.

2. Chittka, L., Skorupski, P., and Raine, N.E. (2009). Speed-accuracy tradeoffs in animal decision making. Trends Ecol. Evol. 24, 400-407.

3. Carland, M.A., Thura, D., and Cisek, P. (2019). The Urge to Decide and Act: Implications for Brain Function and Dysfunction. Neuroscientist 25, 491-511.

4. Frank, M.J., Samanta, J., Moustafa, A.A., and Sherman, S.J. (2007). Hold your horses: Impulsivity, deep brain stimulation, and medication in Parkinsonism. Science (80-. ). 318, 1309-1312.

5. Manohar, S.G., Chong, T.T.J., Apps, M.A.J., Batla, A., Stamelou, M., Jarman, P.R., Bhatia, K.P., and Husain, M. (2015). Reward Pays the Cost of Noise Reduction in Motor and Cognitive Control. Curr. Biol. 25, 1707-1716.

6. Kertzman, S., Vainder, M., Vishne, T., Aizer, A., Kotler, M., and Dannon, P.N. (2010). Speed-accuracy tradeoff in decision-making performance among pathological gamblers. Eur. Addict. Res. 16, 23-30.

7. Schall, J.D. (2019). Accumulators, Neurons, and Response Time. Trends Neurosci. 42, 848-860.

8. Standage, D., Blohm, G., and Dorris, M.C. (2014). On the neural implementation of the speed-accuracy trade-off. Front. Neurosci. 8.

9. Bogacz, R., Wagenmakers, E.J., Forstmann, B.U., and Nieuwenhuis, S. (2010). The neural basis of the speed-accuracy tradeoff. Trends Neurosci. 33, 10-16.

10. Alamia, A., Zénon, A., VanRullen, R., Duque, J., and Derosiere, G. (2019). Implicit visual cues tune oscillatory motor activity during decision-making. Neuroimage 186, 424-436.

11. Cisek, P., and Pastor-Bernier, A. (2014). On the challenges and mechanisms of embodied decisions. Philos. Trans. R. Soc. B Biol. Sci. 369, 2013047920130479.

12. Derosiere, G., Klein, P.A., Nozaradan, S., Zénon, A., Mouraux, A., and Duque, J. (2018). Visuomotor correlates of conflict expectation in the context of motor decisions. J. Neurosci. 38, 9486-9504.

13. Derosiere, G., Vassiliadis, P., Demaret, S., Zénon, A., and Duque, J. (2017). Learning stage-dependent effect of $\mathrm{M} 1$ disruption on value-based motor decisions. Neuroimage 162, 173-185.

14. Derosiere, G., Zénon, A., Alamia, A., and Duque, J. (2017). Primary motor cortex contributes to the implementation of implicit value-based rules during motor decisions. Neuroimage 146, 1115-1127.

15. Gao, Z., Davis, C., Thomas, A.M., Economo, M.N., Abrego, A.M., Svoboda, K., De Zeeuw, C.I., and Li, N. (2018). A cortico-cerebellar loop for motor planning. Nature 563, 113-116. 
16. Yoo, S.B.M., and Hayden, B.Y. (2018). Economic Choice as an Untangling of Options into Actions. Neuron 99, 434-447.

17. Cisek, P. (2007). Cortical mechanisms of action selection: The affordance competition hypothesis. Philos. Trans. R. Soc. B Biol. Sci. 362, 1585-1599.

18. Reppert, T.R., Servant, M., Heitz, R.P., and Schall, J.D. (2018). Neural mechanisms of speed-accuracy tradeoff of visual search: Saccade vigor, the origin of targeting errors, and comparison of the superior colliculus and frontal eye field. J. Neurophysiol. 120, 372-384.

19. Herz, D.M., Tan, H., Brittain, J.S., Fischer, P., Cheeran, B., Green, A.L., Fitzgerald, J., Aziz, T.Z., Ashkan, K., Little, S., et al. (2017). Distinct mechanisms mediate speed-accuracy adjustments in cortico-subthalamic networks. Elife 6, $1-25$.

20. Murphy, P.R., Boonstra, E., and Nieuwenhuis, S. (2016). Global gain modulation generates time-dependent urgency during perceptual choice in humans. Nat. Commun. 7, 1-14.

21. Spieser, L., Kohl, C., Forster, B., Bestmann, S., and Yarrow, K. (2018). Neurodynamic evidence supports a forced-excursion model of decision-making under speed/accuracy instructions. eNeuro 5, 1-16.

22. Steinemann, N.A., O'Connell, R.G., and Kelly, S.P. (2018). Decisions are expedited through multiple neural adjustments spanning the sensorimotor hierarchy. Nat. Commun. 9.

23. Thura, D., Guberman, G., and Cisek, P. (2017). Trial-to-trial adjustments of speed-accuracy trade-offs in premotor and primary motor cortex. J. Neurophysiol. 117, 665-683.

24. Thura, D., and Cisek, P. (2017). The Basal Ganglia Do Not Select Reach Targets but Control the Urgency of Commitment. Neuron 95, 1160-1170.e5.

25. Thura, D., and Cisek, P. (2016). Modulation of Premotor and Primary Motor Cortical Activity during Volitional Adjustments of Speed-Accuracy Trade-Offs. J. Neurosci. 36, 938-956.

26. Heitz, R.P., and Schall, J.D. (2012). Neural Mechanisms of Speed-Accuracy Tradeoff. Neuron 76, 616-628.

27. Hanks, T.D., Kiani, R., and Shadlen, M.N. (2014). A neural mechanism of speedaccuracy tradeoff in macaque area LIP. Elife 2014, 1-17.

28. van Maanen, L., Brown, S.D., Eichele, T., Wagenmakers, E.J., Ho, T., Serences, J., and Forstmann, B.U. (2011). Neural correlates of trial-to-trial fluctuations in response caution. J. Neurosci. 31, 17488-17495.

29. Forstmann, B.U., Dutilh, G., Brown, S., Neumann, J., Von Cramon, D.Y., Ridderinkhof, K.R., and Wagenmakers, E.J. (2008). Striatum and pre-SMA facilitate decision-making under time pressure. Proc. Natl. Acad. Sci. U. S. A. 105, 17538-17542.

30. Herz, D.M., Zavala, B.A., Bogacz, R., and Brown, P. (2016). Neural Correlates 
of Decision Thresholds in the Human Subthalamic Nucleus. Curr. Biol. 26, 916920.

31. Heitz, R.P., and Schall, J.D. (2012). Neural Mechanisms of Speed-Accuracy Tradeoff. Neuron 76, 616-628.

32. Eckhoff, P., Wong-Lin, K.F., and Holmes, P. (2009). Optimality and robustness of a biophysical decision-making model under norepinephrine modulation. J. Neurosci. 29, 4301-4311.

33. Hauser, T.U., Moutoussis, M., Purg, N., Dayan, P., and Dolan, R.J. (2018). Betablocker propranolol modulates decision urgency during sequential information gathering. J. Neurosci. 38, 7170-7178.

34. Ratcliff, R., Smith, P.L., Brown, S.D., and McKoon, G. (2016). Diffusion Decision Model: Current Issues and History. Trends Cogn. Sci. 20, 260-281.

35. Reddi, B.A.J., and Carpenter, R.H.S. (2000). The influence of urgency on decision time. Nat. Neurosci. 3, 827-830.

36. Leon, M.I., and Shadlen, M.N. (2003). Representation of time by neurons in the posterior parietal cortex of the Macaque. Neuron 38, 317-327.

37. Gold, J.I., and Shadlen, M.N. (2007). The Neural Basis of Decision Making.

38. Mazurek, M.E., Roitman, J.D., Ditterich, J., and Shadlen, M.N. (2003). A Role for Neural Integrators in Perceptual Decision Making. Cereb. Cortex 13, 1257-1269.

39. Kelly, S.P., and O'Connell, R.G. (2015). The neural processes underlying perceptual decision making in humans: Recent progress and future directions. J. Physiol. Paris 109, 27-37.

40. Ratcliff, R., and McKoon, G. (2008). The diffusion decision model: Theory and data for two-choice decision tasks. Neural Comput. 20, 873-922.

41. Ratcliff, R. (2004). A Comparison of Sequential Sampling Models for Two-Choice Reaction Time Roger Ratcliff Northwestern University Philip L . Smith University of Melbourne. Psychol. Rev. 111, 1-101.

42. Smith, P.L., and Ratcliff, R. (2004). Psychology and neurobiology of simple decisions. Trends Neurosci. 27, 161-168.

43. Ratcliff, R. (1985). Theoretical interpretations of the speed and accuracy of positive and negative responses. Psychol. Rev. 92, 212-25.

44. Derosiere, G., Vassiliadis, P., and Duque, J. (2020). Advanced TMS approaches to probe corticospinal excitability during action preparation. Neuroimage 213, 116746.

45. Thura, D., Beauregard-Racine, J., Fradet, C.W., and Cisek, P. (2012). Decision making by urgency gating: Theory and experimental support. J. Neurophysiol. 108, 2912-2930.

46. Di Lazzaro, V., Rothwell, J., and Capogna, M. (2018). Noninvasive Stimulation of the Human Brain: Activation of Multiple Cortical Circuits. Neuroscientist 24, 246-260. 
47. Carland, M.A., Marcos, E., Thura, D., and Cisek, P. (2016). Evidence against perfect integration of sensory information during perceptual decision making. J. Neurophysiol. 115, 915-930.

48. Willett, F.R., Deo, D.R., Avansino, D.T., Rezaii, P., Hochberg, L.R., Henderson, J.M., and Shenoy, K. V. (2020). Hand Knob Area of Premotor Cortex Represents the Whole Body in a Compositional Way. Cell 181, 396-409.e26.

49. Labruna, L., Tischler, C., Cazares, C.C., Greenhouse, I., Duque, J., Lebon, F., and Ivry, R.B. (2019). Planning face, hand, and leg movements: Anatomical constraints on preparatory inhibition. J. Neurophysiol. 121, 1609-1620.

50. Derosiere, G., and Duque, J. (2020). Tuning the Corticospinal System: How Distributed Brain Circuits Shape Human Actions. Neuroscientist.

51. Bestmann, S., and Duque, J. (2016). Transcranial Magnetic Stimulation: Decomposing the Processes Underlying Action Preparation. Neuroscientist 22, 392-405.

52. Cisek, P., Puskas, G.A., and El-Murr, S. (2009). Decisions in changing conditions: The urgency-gating model. J. Neurosci. 29, 11560-11571.

53. Oram, M.W. (2011). Visual stimulation decorrelates neuronal activity. J. Neurophysiol. 105, 942-957.

54. Mitchell, J.F., Sundberg, K.A., and Reynolds, J.H. (2009). Spatial Attention Decorrelates Intrinsic Activity Fluctuations in Macaque Area V4. Neuron 63, 879-888.

55. Tetzlaff, T., Helias, M., Einevoll, G.T., and Diesmann, M. (2012). Decorrelation of Neural-Network Activity by Inhibitory Feedback. PLoS Comput Biol 8, 1002596.

56. Morita, K., Kalra, R., Aihara, K., and Robinson, H.P.C. (2008). Recurrent synaptic input and the timing of gamma-frequency-modulated firing of pyramidal cells during neocortical "UP" states. J. Neurosci. 28, 1871-1881.

57. Lytton, W.W., and Sejnowski, T.J. (1991). Simulations of cortical pyramidal neurons synchronized by inhibitory interneurons. J. Neurophysiol. 66, 10591079.

58. Moore, G.P., Segundo, J.P., Perkel, D.H., and Levitan, H. (1970). Statistical Signs of Synaptic Interaction in Neurons. Biophys. J. 10, 876-900.

59. Mitchell, S.J., and Silver, R.A. (2003). Shunting inhibition modulates neuronal gain during synaptic excitation. Neuron 38, 433-445.

60. Derosiere, G., Thura, D., Cisek, P., and Duque, J. (2021). Trading accuracy for speed over the course of a decision. bioRxiv.

61. Derosiere, G., Thura, D., Cisek, P., and Duque, J. (2019). Motor cortex disruption delays motor processes but not deliberation about action choices. J. Neurophysiol. 122, 1566-1577.

62. Drugowitsch, J., Moreno-Bote, R.N., Churchland, A.K., Shadlen, M.N., and Pouget, A. (2012). The cost of accumulating evidence in perceptual decision 
making. J. Neurosci. 32, 3612-3628.

63. Ditterich, J. (2006). Evidence for time-variant decision making. Eur. J. Neurosci. 24, 3628-3641.

64. Ammann, C., Guida, P., Caballero-Insaurriaga, J., Pineda-Pardo, J.A., Oliviero, A., and Foffani, G. (2020). A framework to assess the impact of number of trials on the amplitude of motor evoked potentials. Sci. Rep. 10,1-15.

65. Labruna, L., Fern Andez-Del-Olmo, M., and Ivry, R.B. (2010). Comparison of different baseline conditions in evaluating factors that influence motor cortex excitability. Brain Stimul.

66. Morey, R.D., and Rouder, J.N. (2011). Bayes Factor Approaches for Testing Interval Null Hypotheses. Psychol. Methods 16, 406-419.

67. Bishara, A.J., and Hittner, J.B. (2012). Testing the significance of a correlation with nonnormal data: Comparison of Pearson, Spearman, transformation, and resampling approaches. Psychol. Methods 17, 399-417.

68. Fisher, R. (1921). On the "Probable Error" of a Coefficient of Correlation Deduced from a Small Sample. | Semantic Scholar. Metron 1, 1-32.

69. Churchland, M.M., Santhanam, G., and Shenoy, K. V (2006). Preparatory activity in premotor and motor cortex reflects the speed of the upcoming reach. $J$. Neurophysiol. 96, 3130-3146.

70. Spieser, L., Servant, M., Hasbroucq, T., and Burle, B. (2017). Beyond decision! Motor contribution to speed-accuracy trade-off in decision-making. Psychon. Bull. Rev. 24, 950-956.

71. Hasegawa, M., Majima, K., Itokazu, T., Maki, T., Albrecht, U.R., Castner, N., Izumo, M., Sohya, K., Sato, T.K., Kamitani, Y., et al. (2017). Selective Suppression of Local Circuits during Movement Preparation in the Mouse Motor Cortex. Cell Rep. 18, 2676-2686.

72. Bostan, A.C., and Strick, P.L. (2018). The basal ganglia and the cerebellum: Nodes in an integrated network. Nat. Rev. Neurosci. 19, 338-350.

73. Sohn Mark Hallett, Y.H. (2004). Surround inhibition in human motor system. Exp Brain Res 158, 397-404.

74. Beck, S., Richardson, S.P., Shamim, E.A., Dang, N., Schubert, M., and Hallett, M. (2008). Short intracortical and surround inhibition are selectively reduced during movement initiation in focal hand dystonia. J. Neurosci. 28, 10363-10369.

75. Beck, S., and Hallett, M. (2011). Surround inhibition in the motor system. Exp. Brain Res. 210, 165-172.

76. Poston, B., Kukke, S.N., Paine, R.W., Francis, S., and Hallett, M. (2012). Cortical silent period duration and its implications for surround inhibition of a hand muscle. Eur. J. Neurosci. 36, 2964-2971.

77. Duque, J., Greenhouse, I., Labruna, L., and Ivry, R.B. (2017). Physiological Markers of Motor Inhibition during Human Behavior. Trends Neurosci. 40, 219236. 
78. Greenhouse, I., Sias, A., Labruna, L., and Ivry, R.B. (2015). Nonspecific inhibition of the motor system during response preparation. J. Neurosci. 35, 10675-10684.

79. Quoilin, C., and Derosiere, G. (2015). Global and specific motor inhibitory mechanisms during action preparation. J. Neurosci. 35, 16297-16299.

80. Derosiere, G., and Duque, J. (2020). Tuning the Corticospinal System: How Distributed Brain Circuits Shape Human Actions. Neuroscientist 26.

81. Zohary, E., Shadlen, M.N., and Newsome, W.T. (1994). Correlated neuronal discharge rate and its implications for psychophysical performance. Nature 370, 140-143.

82. Bair, W., Zohary, E., and Newsome, W.T. (2001). Correlated firing in macaque visual area MT: Time scales and relationship to behavior. J. Neurosci. 21, 16761697.

83. Shadlen, M.N., and Newsome, W.T. (1998). The variable discharge of cortical neurons: Implications for connectivity, computation, and information coding. J. Neurosci. 18, 3870-3896.

84. Kohn, A., and Smith, M.A. (2005). Stimulus dependence of neuronal correlation in primary visual cortex of the macaque. J. Neurosci. 25, 3661-3673.

85. Cisek, P., and Kalaska, J.F. (2005). Neural correlates of reaching decisions in dorsal premotor cortex: Specification of multiple direction choices and final selection of action. Neuron 45, 801-814.

86. Alamia, A., Zénon, A., VanRullen, R., Duque, J., and Derosiere, G. (2019). Implicit visual cues tune oscillatory motor activity during decision-making. Neuroimage 186.

87. Pezzulo, G., and Cisek, P. (2016). Navigating the Affordance Landscape: Feedback Control as a Process Model of Behavior and Cognition. Trends Cogn. Sci. 20, 414-424.

88. Thura, D., and Cisek, P. (2014). Deliberation and commitment in the premotor and primary motor cortex during dynamic decision making. Neuron 81, 14011416.

89. Wu, Z., Litwin-Kumar, A., Shamash, P., Taylor, A., Axel, R., and Shadlen, M.N. (2020). Context-Dependent Decision Making in a Premotor Circuit. Neuron 106, 316-328.e6.

90. Meister, M.L.R., Hennig, J.A., and Huk, A.C. (2013). Signal multiplexing and single-neuron computations in lateral intraparietal area during decision-making. J. Neurosci. 33, 2254-2267.

91. Roitman, J.D., and Shadlen, M.N. (2002). Response of neurons in the lateral intraparietal area during a combined visual discrimination reaction time task. J. Neurosci. 22, 9475-9489.

92. Bahl, A., and Engert, F. (2020). Neural circuits for evidence accumulation and decision making in larval zebrafish. Nat. Neurosci. 23, 94-102. 
93. Lin, Q., Manley, J., Helmreich, M., Schier, A., Nö Bauer, T., Correspondence, A.V., Schlumm, F., Li, J.M., Robson, D.N., Engert, F., et al. (2019). Cerebellar Neurodynamics Predict Decision Timing and Outcome on the Single-Trial Level In Brief Article Cerebellar Neurodynamics Predict Decision Timing and Outcome on the Single-Trial Level. Cell 180, 536-551.e17.

94. Aston-Jones, G., and Cohen, J.D. (2005). An integrative theory of locus coeruleus-norepinephrine function: Adaptive gain and optimal performance. Annu. Rev. Neurosci. 28, 403-450.

95. Joshi, S., and Gold, J.I. (2020). Pupil Size as a Window on Neural Substrates of Cognition. Trends Cogn. Sci. 24, 466-480.

96. Reynaud, A.J., Saleri Lunazzi, C., and Thura, D. (2020). Humans sacrifice decision-making for action execution when a demanding control of movement is required. J. Neurophysiol. 124, 497-509.

97. Thura, D. (2020). Decision urgency invigorates movement in humans. Behav. Brain Res. 382, 112477.

98. Thura, D., Cos, I., Trung, J., and Cisek, P. (2014). Context-dependent urgency influences speed-accuracy trade-offs in decision-making and movement execution. J. Neurosci. 34, 16442-16454.

99. Codol, O., Holland, P.J., Manohar, S.G., and Galea, J.M. (2020). Reward-based improvements in motor control are driven by multiple error-reducing mechanisms. J. Neurosci. 40, 3604-3620.

100. Vassiliadis, P., and Derosiere, G. (2020). Selecting and executing actions for rewards. J. Neurosci. 40, 6474-6476.

101. Derosiere, G., Billot, M., Ward, E.T., and Perrey, S. (2015). Adaptations of motor neural structures' activity to lapses in attention. Cereb. Cortex 25, 66-74.

102. Schmidt, C., Peigneux, P., Muto, V., Schenkel, M., Knoblauch, V., Münch, M., De Quervain, D.J.F., Wirz-Justice, A., and Cajochen, C. (2006). Encoding difficulty promotes postlearning changes in sleep spindle activity during napping. J. Neurosci. 26, 8976-8982.

103. Gaggioni, G., Ly, J.Q.M., Muto, V., Chellappa, S.L., Jaspar, M., Meyer, C., Delfosse, T., Vanvinckenroye, A., Dumont, R., Coppieters 't Wallant, D., et al. (2019). Age-related decrease in cortical excitability circadian variations during sleep loss and its links with cognition. Neurobiol. Aging 78, 52-63.

104. Vassiliadis, P., Grandjean, J., Derosiere, G., de Wilde, Y., Quemener, L., and Duque, J. (2018). Using a double-coil TMS protocol to assess preparatory inhibition bilaterally. Front. Neurosci. 12, 139.

105. Grandjean, J., Derosiere, G., Vassiliadis, P., Quemener, L., Wilde, Y. de, and Duque, J. (2018). Towards assessing corticospinal excitability bilaterally: Validation of a double-coil TMS method. J. Neurosci. Methods 293, 162-168.

106. Arai, N., Müller-Dahlhaus, F., Murakami, T., Bliem, B., Lu, M.K., Ugawa, Y., and Ziemann, U. (2011). State-dependent and timing-dependent bidirectional associative plasticity in the human SMA-M1 network. J. Neurosci. 31, 15376- 
15383.

107. Grandjean, J., and Duque, J. (2020). A TMS study of preparatory suppression in binge drinkers. Neurolmage Clin. 28.

108. Vandermeeren, Y., Davare, M., Duque, J., and Olivier, E. (2009). Reorganization of cortical hand representation in congenital hemiplegia. Eur. J. Neurosci. 29, 845-854.

109. Deng, Z. De, Lisanby, S.H., and Peterchev, A. V. (2014). Coil design considerations for deep transcranial magnetic stimulation. Clin. Neurophysiol. $125,1202-1212$.

110. Laczó, B., Antal, A., Rothkegel, H., and Paulus, W. (2014). Increasing human leg motor cortex excitability by transcranial high frequency random noise stimulation. Restor. Neurol. Neurosci. 32, 403-410.

111. Niehaus, L., Meyer, B.U., and Weyh, T. (2000). Influence of pulse configuration and direction of coil current on excitatory effects of magnetic motor cortex and nerve stimulation. Clin. Neurophysiol. 111, 75-80.

112. Rossini, P.M., Barker, A.T., Berardelli, A., Caramia, M.D., Caruso, G., Cracco, R.Q., Dimitrijević, M.R., Hallett, M., Katayama, Y., Lücking, C.H., et al. (1994). Non-invasive electrical and magnetic stimulation of the brain, spinal cord and roots: basic principles and procedures for routine clinical application. Report of an IFCN committee. Electroencephalogr. Clin. Neurophysiol. 91, 79-92.

113. Donner, T.H., Siegel, M., Fries, P., and Engel, A.K. (2009). Buildup of ChoicePredictive Activity in Human Motor Cortex during Perceptual Decision Making. Curr. Biol. 19, 1581-1585.

114. Gould, I.C., Nobre, A.C., Wyart, V., and Rushworth, M.F.S. (2012). Effects of decision variables and intraparietal stimulation on sensorimotor oscillatory activity in the human brain. J. Neurosci. 32, 13805-13818.

115. Wyart, V., de Gardelle, V., Scholl, J., and Summerfield, C. (2012). Rhythmic Fluctuations in Evidence Accumulation during Decision Making in the Human Brain. Neuron 76, 847-858.

116. Vassiliadis, P., Derosiere, G., Grandjean, J., and Duque, J. (2020). Motor training strengthens corticospinal suppression during movement preparation. bioRxiv, 2020.02.14.948877.

117. Duque, J., and Ivry, R.B. (2009). Role of corticospinal suppression during motor preparation. Cereb. Cortex 19, 2013-2024.

118. Liu, X., Zhu, X.H., Qiu, P., and Chen, W. (2012). A correlation-matrix-based hierarchical clustering method for functional connectivity analysis. J. Neurosci. Methods 211, 94-102.

119. Niu, H., and He, Y. (2014). Resting-state functional brain connectivity: Lessons from functional near-infrared spectroscopy. Neuroscientist 20, 173-188.

120. Yordanova, Y.N., Cochereau, J., Duffau, H., and Herbet, G. (2019). Combining resting state functional $\mathrm{MRI}$ with intraoperative cortical stimulation to map the 
mentalizing network. Neuroimage 186, 628-636.

121. Cohen, J. (2013). Statistical Power Analysis for the Behavioral Sciences (Routledge).

122. Miles, J.N., and Shevlin, M. (2001). Applying regression \& correlation : a guide for students and researchers. undefined.

123. Kass, R.E., and Raftery, A.E. (1995). Bayes Factors. J. Am. Stat. Assoc. 90, 773. 